\title{
Sensitivity of Spin Parameters to Uncertainties of the Aircraft Aerodynamic Model
}

\author{
Dominique Farcy* \\ ONERA-LMFL, F-59000 Lille, France \\ and \\ Alexander N. Khrabrov‡ and Maria E. Sidoryuk辛 \\ TsAGI, 140180, Zhukovsky, Russia \\ https://doi.org/10.2514/1.C035882
}

\begin{abstract}
Reliable prediction of aircraft poststalled dynamics is entirely dependent on the adequacy of the aerodynamic model. A mathematical model of aerodynamic characteristics of a light aircraft is developed for a wide range of angles of attack, sideslip, and angular rates. It is augmented by a model of uncertainties based on a set of repeated measurements of static aerodynamic characteristics. The effect of aerodynamic uncertainties on steady-state and oscillatory spin modes is directly calculated using a continuation on a parameter technique. The influence of aerodynamic uncertainties on spin entry/recovery is also analyzed.
\end{abstract}

\begin{tabular}{|c|c|c|}
\hline$b$ & $=$ & aircraft span, $\mathrm{m}$ \\
\hline$C_{l}, C_{m}, C_{n}$ & $=$ & roll, pitch, and yaw moment coefficients \\
\hline$C_{X}, C_{Y}, C_{Z}$ & $=$ & $\begin{array}{l}\text { force coefficients in the } x, y \text {, and } z \\
\text { directions }\end{array}$ \\
\hline $\bar{c}$ & $=$ & mean aerodynamic chord, $\mathrm{m}$ \\
\hline$I=\operatorname{diag}\left(I_{x}, I_{y}, I_{z}\right)$ & $=$ & matrix of moment of inertia, $\mathrm{kg} \cdot \mathrm{m}^{2}$ \\
\hline$m$ & $=$ & mass, $\mathrm{kg}$ \\
\hline$p, q, r$ & $=$ & body roll, pitch, and yaw rates, $\mathrm{rad} / \mathrm{s}$ \\
\hline$p_{a}, q_{a}, r_{a}$ & $=$ & angular rates in wind axes, $\mathrm{rad} / \mathrm{s}$ \\
\hline $\bar{p}, \bar{q}, \bar{r}$ & $=$ & $\begin{array}{l}\text { nondimensional roll, pitch, and yaw rates } \\
\text { in body axes }\end{array}$ \\
\hline $\bar{p}_{a}, \bar{q}_{a}, \bar{r}_{a}$ & $=$ & $\begin{array}{l}\text { nondimensional angular rates in wind } \\
\text { axes }\end{array}$ \\
\hline$S$ & $=$ & reference wing area, $\mathrm{m}^{2}$ \\
\hline$V$ & $=$ & velocity, $\mathrm{m} / \mathrm{s}$ \\
\hline$\alpha, \beta$ & $=$ & angles of attack and sideslip, deg \\
\hline$\delta=\left(\delta_{e}, \delta_{a}, \delta_{r}\right)$ & $=$ & $\begin{array}{l}\text { elevator, aileron, and rudder deflections, } \\
\text { deg }\end{array}$ \\
\hline$\theta$ & $=$ & pitch angle, deg \\
\hline$\phi$ & $=$ & bank angle, deg \\
\hline$\Omega$ & $=$ & aircraft conical rate, $\mathrm{rad} / \mathrm{s}$ \\
\hline$\omega$ & $=$ & nondimensional conical rate \\
\hline
\end{tabular}

\section{Introduction}

$\mathbf{R}$ ELIABLE prediction of aircraft spin, a substantially nonlinear and one of the most dangerous phenomena encountered in flight, is entirely related to the adequacy of the aerodynamic model used for the analysis. The model must be valid in a wide range of angles of attack and sideslip with account of the intensive rotation

Received 17 January 2020; revision received 29 March 2020; accepted for publication 28 April 2020; published online Open Access 29 May 2020. Copyright $\odot 2020$ by the American Institute of Aeronautics and Astronautics, Inc. All rights reserved. All requests for copying and permission to reprint should be submitted to CCC at www.copyright.com; employ the eISSN 1533-3868 to initiate your request. See also AIAA Rights and Permissions www.aiaa.org/randp.

*Engineer-Senior Scientist; CNRS, ONERA, Central Lille, Arts et Metiers ParisTech, FRE 2017-Laboratoire de Mécanique des Fluides de Lille, Kampé de Feriet; dominique.farcy@onera.fr.

${ }^{\dagger}$ Leading Researcher, Flight Dynamics and Control Systems Department, Moscow Region; khrabrov@tsagi.ru.

Leading Researcher, Flight Dynamics and Control Systems Department, Moscow Region; msid@mail.ru. effects. The procedure for developing a heuristic nonlinear aerodynamic model suitable for calculating spin regimes is generally known in the literature. It is usually based on several types of wind-tunnel experiments. Since the experimental data contain different sources of errors, and a heuristic model contains a number of admissions, it is important to investigate sensitivity of calculated bifurcation patterns of critical flight regimes and spin parameters to various uncertainties of the aerodynamic model. In other words, it is important to investigate whether the poststalled aircraft dynamics remains close to the nominal one when the nominal aerodynamic model is perturbed; i.e., estimate the "robustness" of the spin parameters picture to the uncertainties of the aerodynamic model.

The robustness issues for normal flight regimes have been thoroughly studied during the last three decades. A number of methods of robust analysis and synthesis were proposed ( $\mu$ analysis, Lyapunovbased methods, etc.) and supplied by a software [1]. They were intensively used for flight control design and robust stability and performance analysis in many engineering applications. The influence of aerodynamic model uncertainties on critical flight regimes was investigated substantially less, but this is an important issue of adequacy of the aerodynamic models for reliable prediction of poststalled aircraft dynamics.

This work was carried out in the framework of the ONERATsAGI collaborative project titled "Improvement of Representativeness of Models" aimed at the accurate prediction of the aircraft poststalled behavior including spin. The investigations are based on the adequate mathematical modeling of the aircraft's aerodynamic characteristics. The mathematical model of the aerodynamic characteristics of a light aircraft is developed for a wide range of angles of attack, sideslip angles, angular rates, and various controls deflections from ordinary flight to the poststall region. The model is built based on the measurements of aerodynamic efforts in the vertical wind tunnel of ONERA-Lille during static and rotary balanced tests. The generic aerodynamic model is augmented by a model of uncertainties based on a set of repeated measurements of static characteristics of the aircraft with various combinations of test parameters. The results for various values of angle of attack and sideslip, different methods of model suspension, and various flow rates in the wind tunnel are taken into account.

Numerical prediction of spin parameters based on the developed aerodynamic model is performed directly using a technique of continuation on a parameter [2-9] and related methods of bifurcation and stability analysis. The equilibrium and oscillatory spin solutions are calculated for full nonlinear equations of the aircraft motion depending on the parameters that are the deflections of aircraft control surfaces. The calculated results are compared with similar results 
for a reduced system of the third order (angle of attack, sideslip, and angular rate around the velocity vector). The effect of uncertainty of each aerodynamic coefficient and various combinations of uncertainties of coefficients is directly calculated and analyzed for different stationary and oscillatory spin modes. The worst combinations of uncertainties, leading to the largest areas of stable spins, are searched. The emphasis is made on the effects, which are essential for application to analysis of poststalled aircraft behavior; spin entry; and spin recovery.

The paper is arranged as follows. The aerodynamic model structure, its augmentation by uncertainties, and comparison of spin parameters calculated in accordance with the full and approximate equations are described in Sec. II. Sensitivity of spin to damping and uncertainties of static terms of the aerodynamic model, including spin entry/recovery issues, is investigated in Sec. III. Section IV concludes the paper. A preliminary version of this work was presented at the European Conference for Aerospace Sciences (EUCASS) conference [10].

\section{Background}

\section{A. Aerodynamic Model Structure}

The model of aerodynamic characteristics was obtained on the base of a large number of experiments in the ONERA-Lille vertical wind tunnel: static tests in a wide range of angles of attack, sideslip, and conical rotation of the aircraft model near the velocity vector. The investigated aircraft model has a span $2 \mathrm{~m}$, and the wind-tunnel diameter is $4 \mathrm{~m}$. To investigate a total range of angles of attack, two mountings were used: a dorsal mounting for the range of angles of attack of $\alpha \in$ [45 deg, $90 \mathrm{deg}$ ], and a rear sting for the range [ $-45 \mathrm{deg}, 45 \mathrm{deg}]$. They are shown in Fig. 1 . The aerodynamic data are combined from two databases obtained with the two mountings. Measurements on rotary balance were performed with rotation rate about the velocity axis from -600 to $+600 \mathrm{deg} / \mathrm{s}$ under test conditions with $R e=0.83 \times 10^{6}$ and flow velocity of $V=50 \mathrm{~m} / \mathrm{s}(M=0.15)$.

An example of static yaw moment coefficient dependence on angle of attack for a zero sideslip condition is presented in Fig. 2a, demonstrating a high level of uncertainty and a high-level lateral asymmetry due to flow separation on the fuselage and wing. The flow separation leads to a loss of stability of a symmetrical flow state and the appearance of two stable asymmetrical states. Which of these two states is realized depends on a number of unpredictable reasons and can vary from one test to another. For a flow in the wind tunnel, the support has a great influence. It is known [4] that, due to the yaw asymmetry, the right spin modes can significantly differ from the left ones: both in spin parameters and the character of stability. In this paper, we consider only one realization of aerodynamic asymmetry, concentrating on the influence of variations in aerodynamic coefficients around the average value of the chosen asymmetry.

The original ONERA aerodynamic model is essentially nonlinear, and the dimensionless coefficients of the aerodynamic forces and moments have the following structure:

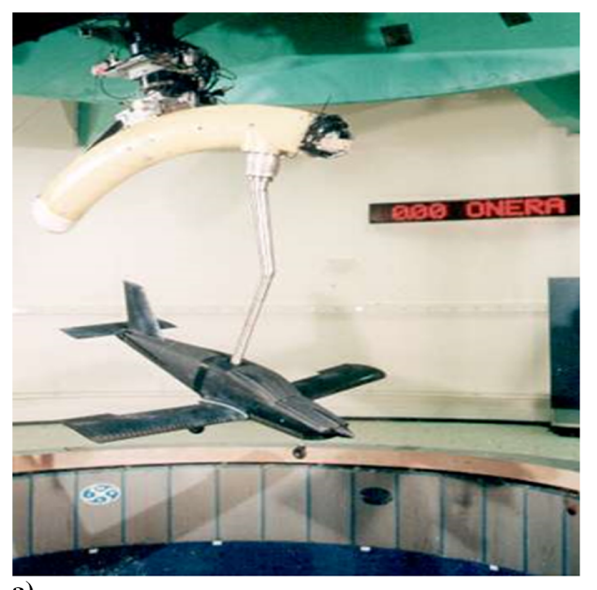

a)

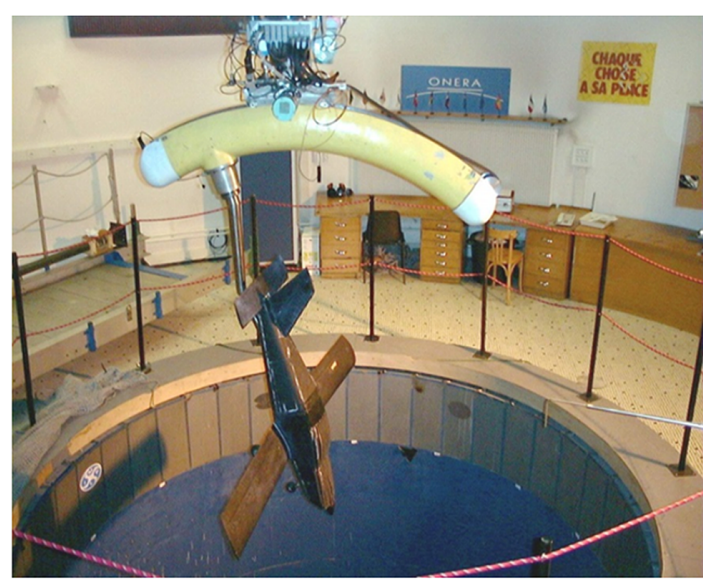

b)

Fig. 1 Vertical wind tunnel and two mountings used for tests: a) dorsal mounting, and b) rear mounting.

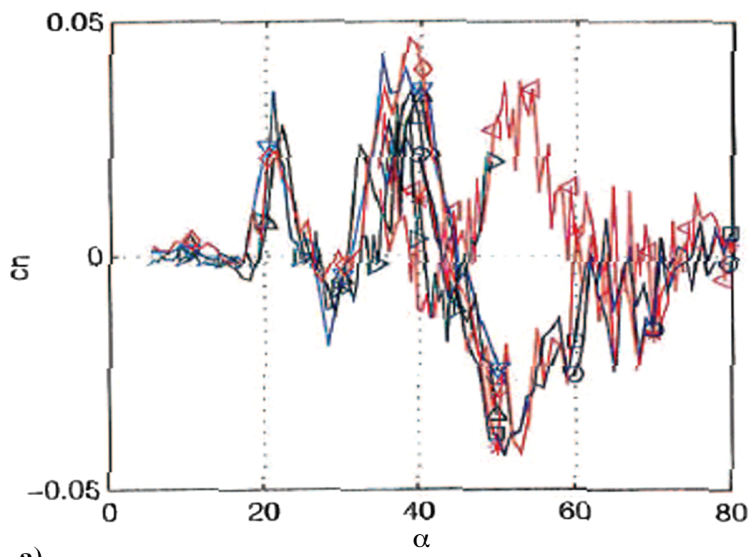

a)

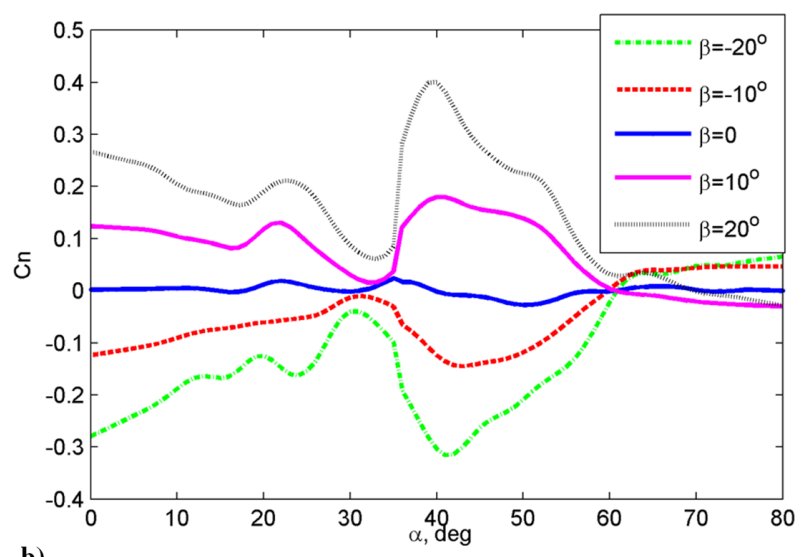

b)

Fig. 2 Static yaw moment coefficient in experiments: a) experiment, and b) modeling. 


$$
\begin{aligned}
C_{X}(\alpha) & =C_{X 0}(\alpha) \\
C_{Y}(\alpha, \beta, \omega, \delta) & =C_{Y 0}(\alpha, \beta)+\Delta C_{Y}(\alpha, \beta, \omega)+\Delta C_{Y}\left(\alpha, \delta_{a}\right)+\Delta C_{Y}\left(\alpha, \delta_{r}\right) \\
C_{Z}(\alpha, \beta, \omega, \delta) & =C_{Z 0}(\alpha, \beta)+\Delta C_{Z}(\alpha, \beta, \omega)+\Delta C_{Z}\left(\alpha, \delta_{e}\right) \\
C_{l}(\alpha, \beta, \omega, \delta) & =C_{l 0}(\alpha, \beta)+\Delta C_{l}(\alpha, \beta, \omega)+\Delta C_{l}\left(\alpha, \delta_{a}\right)+\Delta C_{l}\left(\alpha, \delta_{r}\right) \\
C_{m}(\alpha, \beta, \omega, \delta) & =C_{m 0}(\alpha, \beta)+\Delta C_{m}(\alpha, \beta, \omega)+\Delta C_{m}\left(\alpha, \delta_{e}\right) \\
C_{n}(\alpha, \beta, \omega, \delta) & =C_{n 0}(\alpha, \beta)+\Delta C_{n}(\alpha, \beta, \omega)+\Delta C_{n}\left(\alpha, \delta_{a}\right)+\Delta C_{n}\left(\alpha, \delta_{r}\right)
\end{aligned}
$$

The model of aerodynamic characteristics [Eq. (1)] depends on angles $\alpha$ and $\beta$; deflections of the elevator $\delta_{e}$, aileron $\delta_{a}$, and rudder $\delta_{r}$; and the full nondimensional angular rate of $\omega=\Omega \bar{c} / V$. The nonlinear static dependences $\Delta C_{i 0}(\alpha, \beta) \quad(i=Y, Z, l, m, n)$ and inputs from control surface deflections $\Delta C_{i}\left(\alpha, \delta_{j}\right)(i=Y, l, n, j=$ $a, r)$ and $\Delta C_{i}\left(\alpha, \delta_{e}\right)(i=Z, m)$ are specified through linear interpolated two-dimensional lookup tables. Inputs measured in conical rotation $\Delta C_{i}(\alpha, \beta, \omega)(i=Y, Z, l, m, n)$ are specified through linear interpolated three-dimensional lookup tables. Dependence of $\Delta C_{l}(\alpha, \beta, \omega)$ and $\Delta C_{n}(\alpha, \beta, \omega)$ on angle of attack at $\beta=0$ and several nondimensional angular rate values $\omega=0, \pm 0.05, \pm 0.1$ is shown in Fig. 3.

Lack of dependency of model (1) on angular rate components $p$, $q$, and $r$ separately makes it impossible to adequately predict stability properties of different critical flight regimes. In this case, all the calculated spin modes turned out to be unstable due to lack of sufficient damping in the aerodynamic model $[10,11]$. An unreliable picture of stability of spin modes does not allow us to adequately predict the behavior of the aircraft in case of loss of control. Damping terms in the aerodynamic model can be obtained from the oscillatory coning tests. In the absence of data, an alternative is blending of rotary balance and forced oscillation test data $[9,12-14]$. This approach gives good predictions of the parameters of steady developed spins but fails for the transitional poststall dynamics [14]. Boundaries of the approach are discussed in Ref. [14]. Because of lack of oscillatory coning or forced oscillation test data, the aerodynamic model [Eq. (1)] was supplemented by additional damping terms taken from the available small-amplitude forced oscillation tests in pitch, roll, and yaw for an airplane similar to the ONERA light aircraft [11] using the blending approach $[12,13]$. The modified aerodynamic coefficients with the added damping have the following form:

$$
C_{i}^{d}(\alpha, \beta, \bar{p}, \bar{q}, \bar{r}, \boldsymbol{\delta})=C_{i}\left(\alpha, \beta, \bar{p}_{a}, \boldsymbol{\delta}\right)+C_{i \bar{r}_{a}} \bar{r}_{a}+C_{i \bar{q}_{a}} \bar{q}_{a}, \quad i=l, m, n
$$

where $C_{i}\left(\alpha, \beta, \bar{p}_{a}, \boldsymbol{\delta}\right) \equiv C_{i}(\alpha, \beta, \omega, \boldsymbol{\delta})$ are the dimensionless aerodynamic coefficients in Eq. (1); and $\bar{p}_{a}=p_{a} \bar{c} / V, \bar{q}_{a}=q_{a} \bar{c} / V$, and $\bar{r}_{a}=r_{a} \bar{c} / V$ are the roll, pitch, and yaw rate projections of the dimensionless angular rate on wind axes, respectively, connected with nondimensional body axes angular rates $\bar{p}, \bar{q}$, and $\bar{r}$ as follows:

$$
\begin{aligned}
\bar{p}_{a} & =\bar{p} \cos \alpha \cos \beta+\bar{r} \sin \alpha \cos \beta+\bar{q} \sin \beta \\
\bar{r}_{a} & =\bar{p} \sin \alpha-\bar{r} \cos \alpha \\
\bar{q}_{a} & =-\bar{p} \cos \alpha \sin \beta-\bar{r} \sin \alpha \sin \beta+\bar{q} \cos \beta
\end{aligned}
$$

Here, $\bar{p}_{\text {ฐ }}=\omega$ coincides with the nondimensional angular rate in steady conical rotation in rotary balance tests, and $\bar{r}_{a}$ and $\bar{q}_{a}$ components can be considered as disturbances for pure conical rotation [14]. They are normally small with respect to conical rotation rate $\omega$. The aerodynamic derivatives in the wind-axes system $C_{i \bar{q}_{a}}$ and $C_{i \bar{r}_{a}}, i=l, m, n$ in the coefficients of Eq. (2) are determined by recalculating from body-axis damping derivatives obtained in forced oscillation tests. Dependence of these damping derivatives on angle of attack is shown in Fig. 4. This dependence is nonlinear at moderate and high angles of attack; there exist ranges of antidamping. Therefore, the replacement of these aerodynamic data by the data for a similar aircraft can give only approximate results that, however, are important for understanding the qualitative picture and assessing the sensitivity of spin calculation results to the damping in the aerodynamic model. Results of the simulation of rapid maneuvers and agitated oscillatory spins can be also considered only as qualitative.

The nominal mass/inertia parameters of the aircraft are the following: mass $m=1000 \mathrm{~kg}$; moments of inertia of $I_{x}=1883 \mathrm{~kg} \cdot \mathrm{m}^{2}$, $I_{y}=2205 \mathrm{~kg} \cdot \mathrm{m}^{2}$, and $I_{z}=3356 \mathrm{~kg} \cdot \mathrm{m}^{2}$; and teh center-of-gravity position is $0.20 \bar{c}$.

\section{B. Augmentation of the Aerodynamic Model by Uncertainties}

There is a number of sources of uncertainties of the aerodynamic model: influence of mounting devices used in the wind-tunnel tests, errors caused by extrapolation of aerodynamic data obtained for a scaled model on a real aircraft, a high level of aerodynamic uncertainties caused by flow separation at moderate/high angles of attack in the vicinity of stall, etc. To analyze the influence of uncertainties of the aerodynamic characteristics determined by experiments on calculation of the aircraft's dynamic behavior in the stalling and spin areas, a large number of experiments was conducted in ONERA, and a special technique for reliable averaging of various data was developed. Repeated wind-tunnel measurements of the static characteristics of the aircraft under consideration were performed with various combinations of values of angle of attack and sideslip shown in Fig. 5, with different methods of model suspension, different flow rates in the wind tunnel, etc. Averaged aerodynamic characteristics were obtained by the locally weighted projection regression (LWPR) method [15-17], which is an incremental algorithm for approximation of functions. This allows us to take into account a variety of results when testing in wind tunnels due to different experimental conditions (Reynolds number effects, installation effects, etc.) and calculate the estimate of the reliability of the calculation. The final model is a combination of linear models weighted by Gaussian functions. After averaging, a mean value of aerodynamic asymmetry was retained since it is an important feature of aerodynamics at high
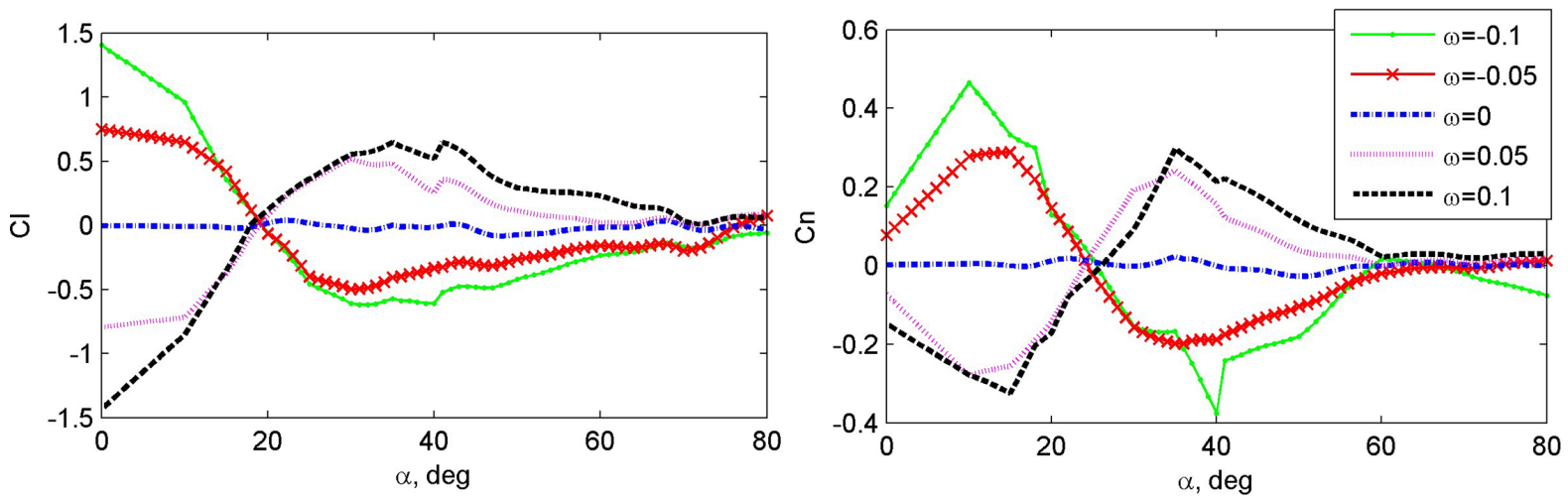

Fig. 3 Roll and yaw moment terms measured in conical rotation at $\beta=0$. 

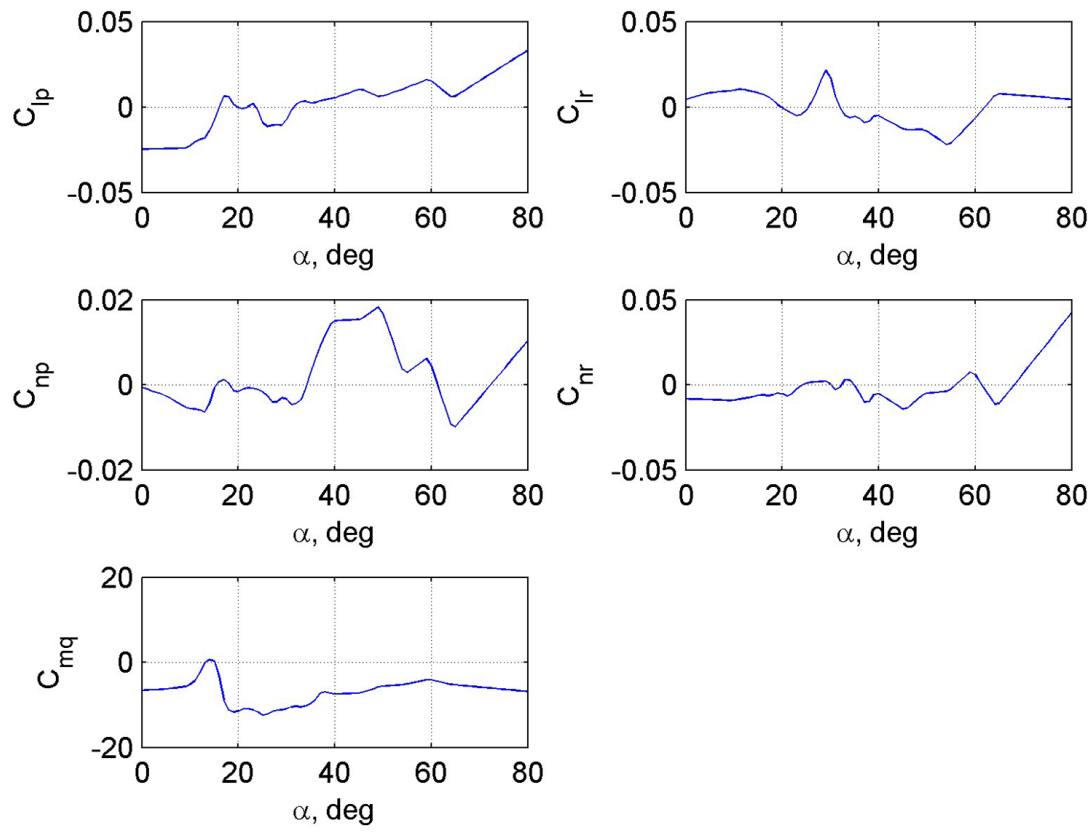

Fig. 4 Damping derivatives versus angle of attack.

angles of attack caused by asymmetric flow separation. An example of the averaged static moment coefficients and the obtained confidence intervals is shown in Fig. 6. Figure 7 shows the dependence of the resulting static aerodynamic moment and side force coefficients on angle of attack and sideslip; the dotted lines show confidence bounds.

\section{Calculation of Spin Parameters, and Comparison with Approxi- mate Approach}

Calculation of spin and other critical regimes is based on a technique of numerical continuation of equilibrium and periodical solutions of nonlinear aircraft dynamics on a parameter [2-9]. The continuation method is a standard prediction-correction method [2]. A usual way to analyze the aircraft spin dynamics is considering the eighth-order autonomous system of motion equations obtained from the full six-degree-of-freedom motion equations in an assumption of fixed altitude [8] using the aerodynamic model Eqs. (1) and (2)]:

$$
\dot{\boldsymbol{x}}=\boldsymbol{F}(\boldsymbol{x}, \boldsymbol{\delta})
$$

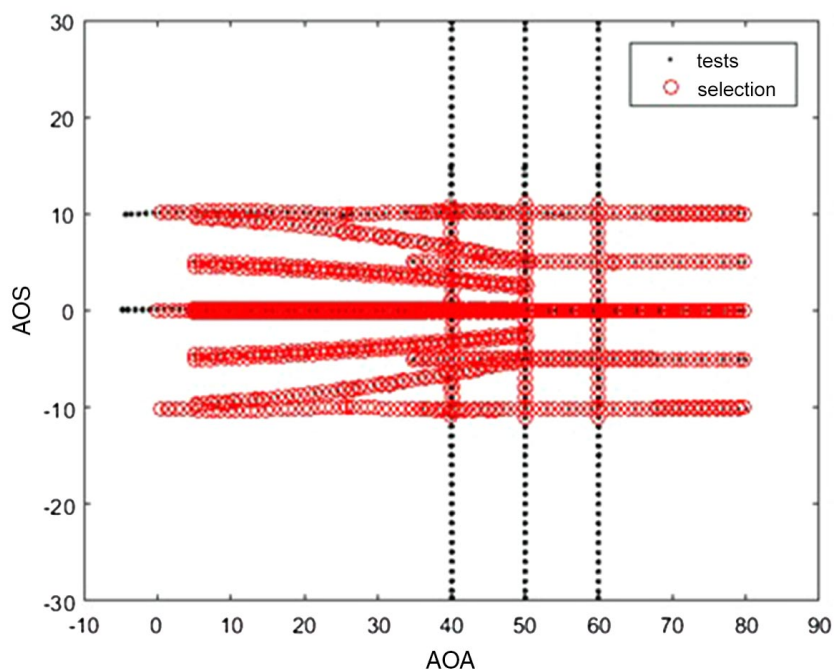

Fig. 5 Training data: angle of attack (AOA) and angle of sideslip (AOS). where the state vector is $x=(V, \alpha, \beta, p, q, r, \theta, \phi)^{\prime} \in R^{8}$, and the control vector is $\boldsymbol{\delta}=\left(\delta_{e}, \delta_{a}, \delta_{r}\right)^{\prime}$. In a procedure of continuation, one of the control surfaces' deflections is used as a parameter, and two others are fixed. Thrust is considered equal to zero. The equilibrium states are defined by the system of algebraic equations:

$$
\boldsymbol{F}(\boldsymbol{x}, \boldsymbol{\delta})=0
$$

When calculating equilibria, their local stability is also analyzed using the linearized system of equations. Continuation of limit cycles or periodical solutions is performed by solving the parameter-dependent boundary-value problem:

$$
x(T, \delta)-x(0, \delta)=0
$$

where the time of period $T$ is one of the unknown variables. At high angles of attack and fast rotation, the equilibrium solutions of system (4) correspond to stationary spin modes, and solutions of Eq. (ㅁ) describe oscillatory spin modes. Calculations are performed by continuation on one of the parameters: elevator deflection, ailerons, or rudder deflection.

Approximate calculation of spin parameters is often performed using reduced third-order equations expressing the balance of aerodynamic and inertial moments acting on the aircraft:

$$
\begin{aligned}
& \frac{\left(I_{y}-I_{z}\right)}{1 / 2 \rho S \bar{c}^{3}} \omega^{2} \sin \alpha \sin \beta \cos \beta+C_{l}(\alpha, \beta, \omega, \delta)=0 \\
& \frac{\left(I_{z}-I_{x}\right)}{1 / 2 \rho S \bar{c}^{3}} \omega^{2} \sin \alpha \cos \alpha \cos ^{2} \beta+C_{m}(\alpha, \beta, \omega, \delta)=0 \\
& \frac{\left(I_{x}-I_{y}\right)}{1 / 2 \rho S \bar{c}^{3}} \omega^{2} \cos \alpha \sin \beta \cos \beta+C_{n}(\alpha, \beta, \omega, \delta)=0
\end{aligned}
$$

These equations are obtained from the full-order motion equations while retaining only the equations for the moments, taking into the aerodynamic moment coefficients in the form of Eq. (1) and the approximate relations $p=\Omega \cos \alpha \cos \beta, q=\Omega \sin \alpha \sin \beta$, and $r=\Omega \sin \alpha \cos \beta$. The solutions of system (6) are used also as initial approximations when calculating spin equilibria of the full-order equations. Note that Eq. (6) contains only the total nondimensional angular rate $c$. An accuracy of approximate solutions is estimated, comparing the results of the continuation of equilibria of the reducedand eighth-order systems considering the same aerodynamic model 

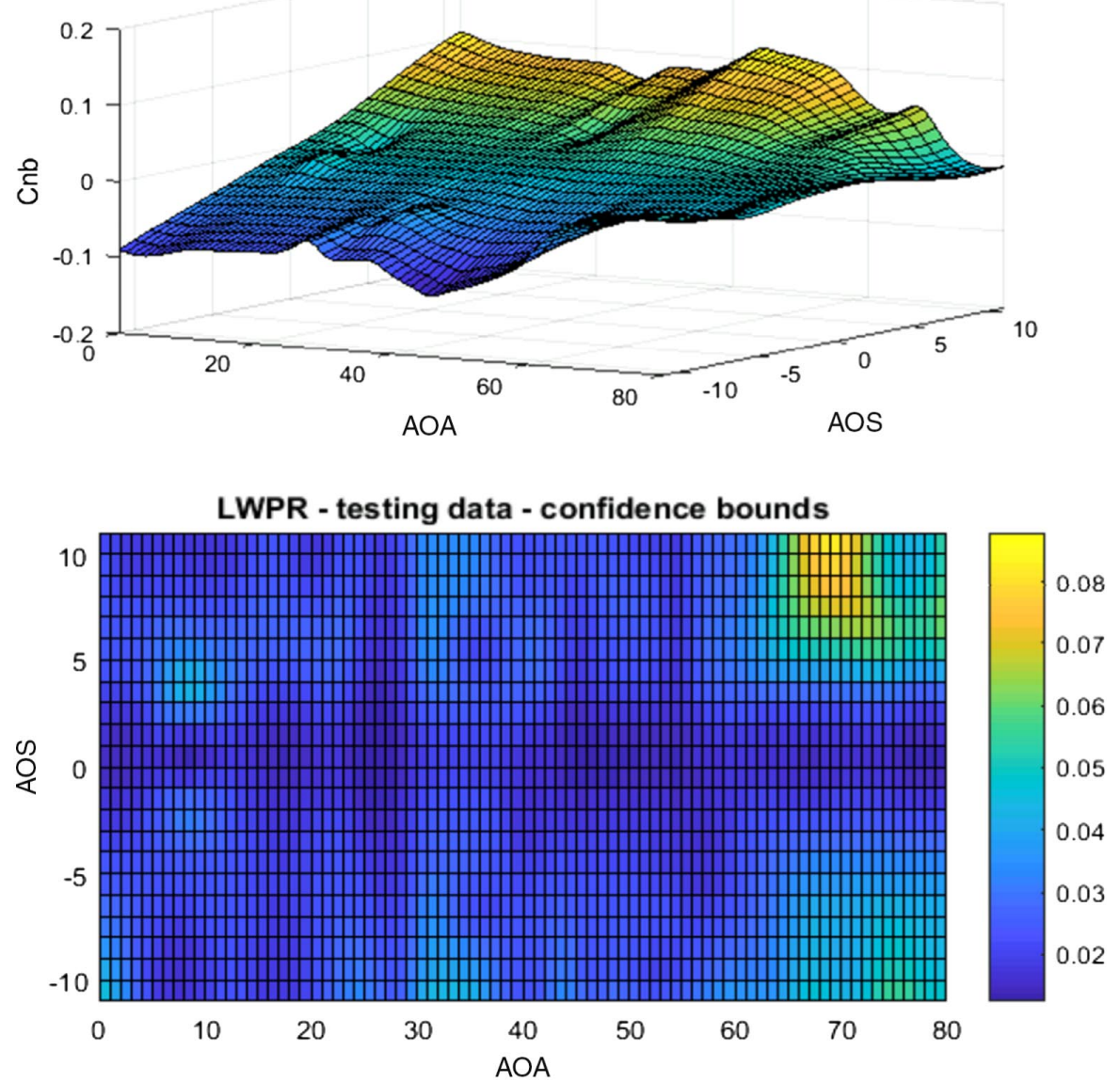

Fig. 6 Nonlinear uncertainties modeling.
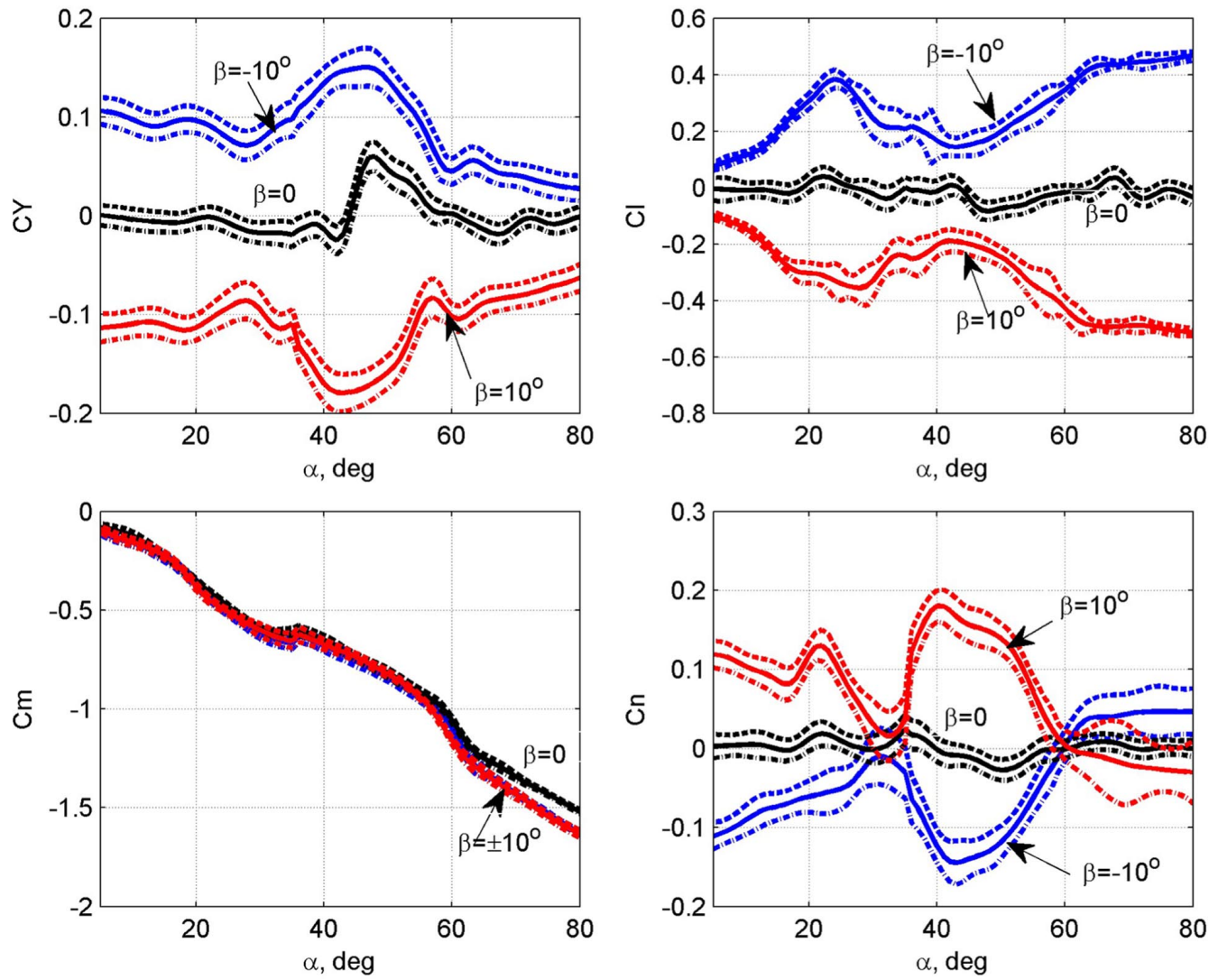

Fig. 7 Static aerodynamic moment coefficients with uncertainty bounds. 

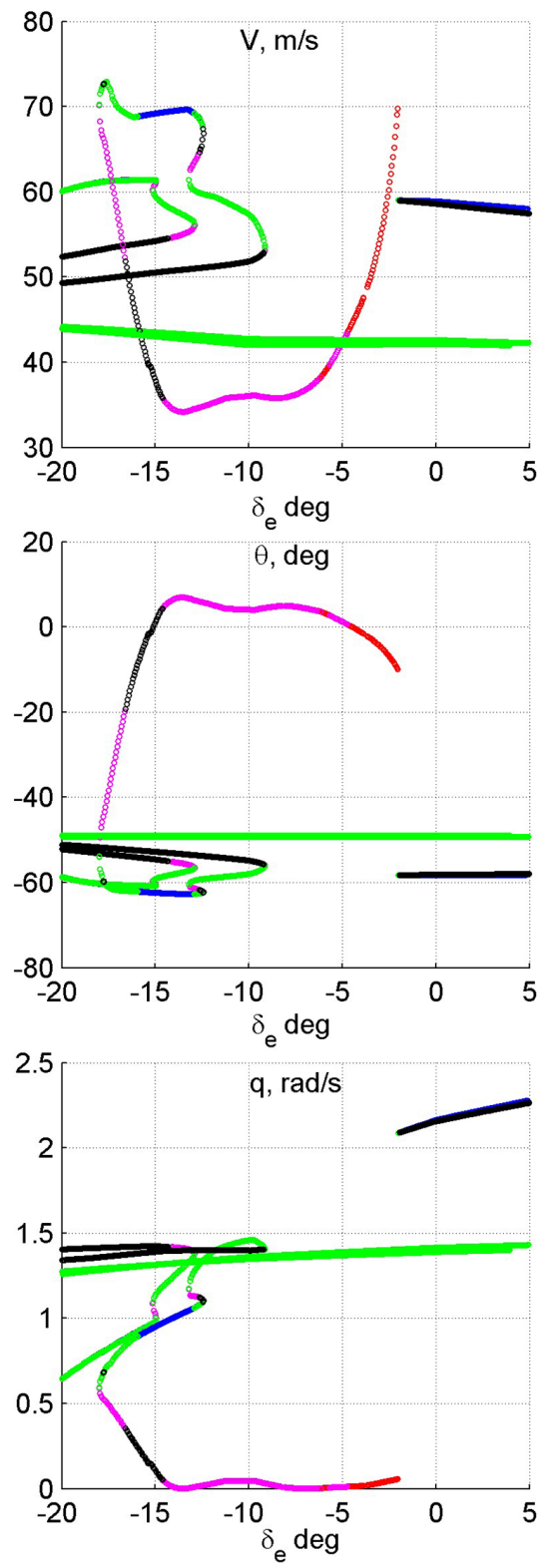
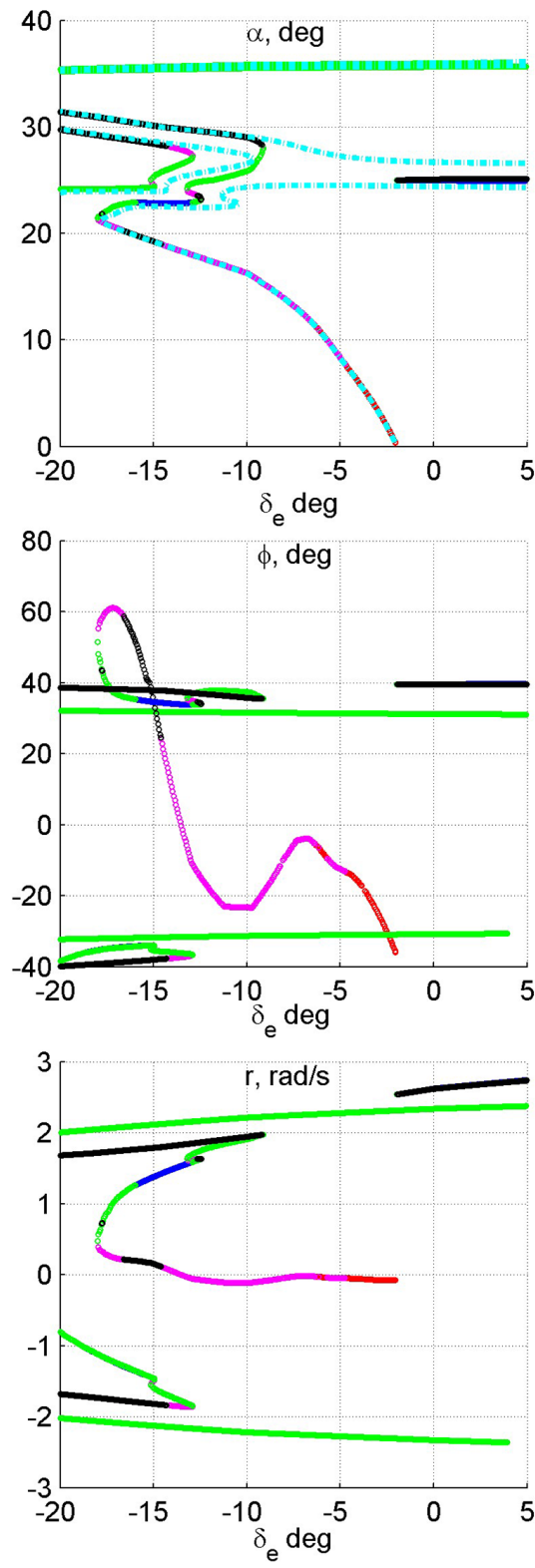
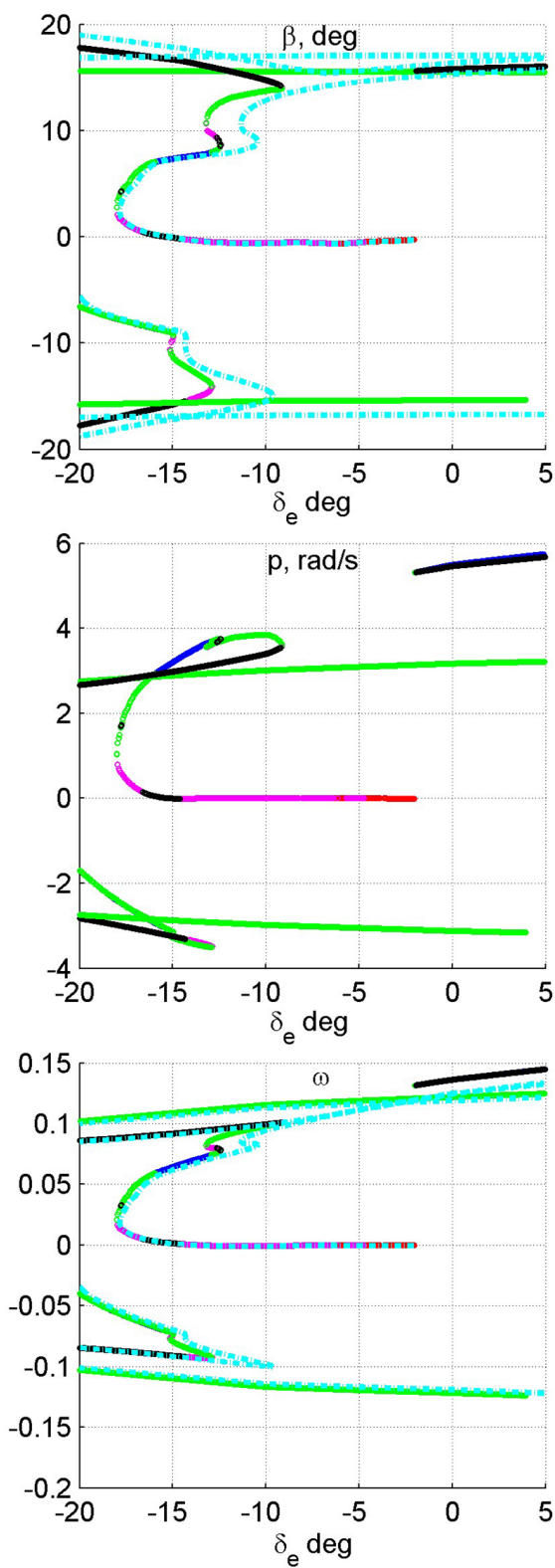

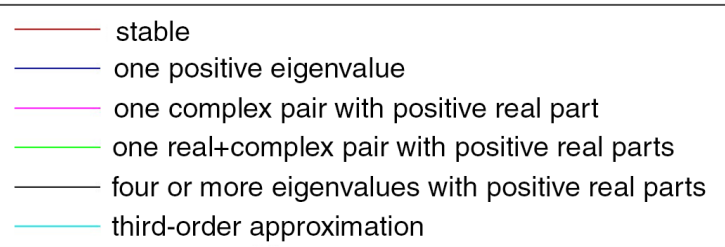

Fig. 8 Comparison of equilibria according to the full and approximate equations: $H=3000 \mathrm{~m}, \delta_{a}=0$, and $\delta_{r}=0$.

[Eq. (1)] without damping terms. The results of such a comparison obtained by continuation on elevator deflection are shown in Fig. 8 (for $\alpha<40 \mathrm{deg}$ ), which describes different stationary solutions of the system, from normal flight regimes to spins. Here, the solutions of reduced system ( $\underline{6}$ ) are marked in cyan. All other colors correspond to the equilibrium solutions of the eighth-order system; they are listed in Fig. 8, depending on the stability properties, related to the number of equilibrium eigenvalues in the right half-plane.

As can be seen from Fig. $\underline{8}$, for most of the range of control deflections, the parameters of equilibria calculated using the approximate and full equations coincide well. However, the range of existence of spins can differ significantly: tuning points in bifurcation diagrams have different coordinates. Moreover, bifurcation diagrams according to the reduced and complete equations can be very similar for one altitude of $H=500 \mathrm{~m}$ and differ significantly for another altitude of $H=3000 \mathrm{~m}$, as seen from Fig. 9. This does not mean that the accuracy of the reduced model is altitude dependent. Slightly above $3000 \mathrm{~m}$, both bifurcation pictures again become very similar. But, the change of the graphs occurs at slightly different altitudes, and this example demonstrates that, if just one approximation is calculated, then an erroneous result can be obtained.

Therefore, the approximate calculation of spin equilibria gives useful information about their parameters but an inaccurate picture of their ranges. The use of complete order equations can give significantly more accurate results. Note that all equilibria with nonzero angular rates are unstable in the absence of additional damping terms. 

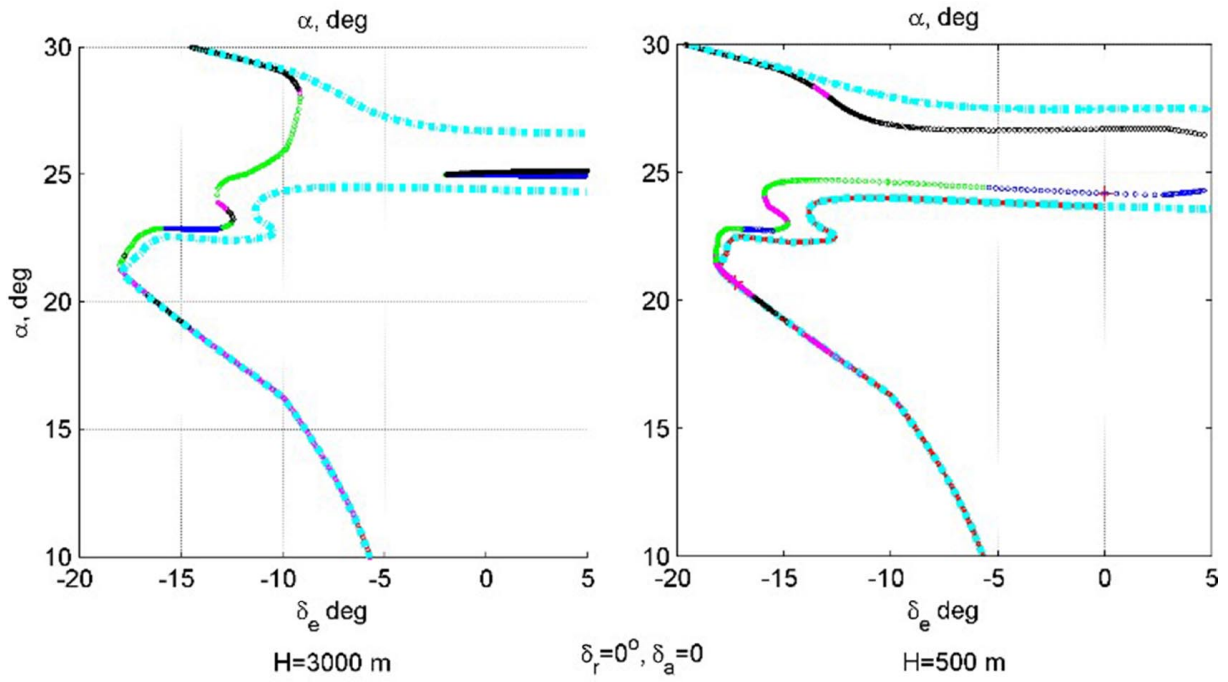

Fig. 9 Comparison of equilibria according to full and reduced equations: different altitudes.

\section{Sensitivity of Spin Parameters to Uncertainties of the Aerodynamic Coefficients}

Spin characteristics substantially depend on the mass/inertia parameters. These issues have been considered by many authors. In particular, one of the analytical criteria for aircraft that focuses on identifying relatively low- $\alpha$ instabilities that can lead to spin onset (departure susceptibility parameter [18]) directly depends on the parameter $I_{x} / I_{z}$. An analytical criterion for spin susceptibility based on the occurrence of saddle-node bifurcation points at high angles of attack and large yaw rates indicating incipient spin [19] directly depends on the parameter $\left(I_{z}-I_{x}\right) / I_{y}$. Changes in inertia (due to fuel consumption) are usually taken into account in the spin analysis for certification of the aircraft, and changes in the picture of spins can be significant. But, we focus only on the effect of variations in the measured aerodynamic data on spin prediction.

\section{A. Influence of Damping Terms}

Comparison of bifurcation diagrams with and without additional damping terms (thick and thin lines, respectively) is shown in Fig. 10 (here and further, only four from the full set of variables are presented). Adding the damping terms results in the appearance of a narrow range of stable spins (marked red) at $\delta_{e} \in[-17.7 \mathrm{deg}$, $-16.8 \mathrm{deg}]: \alpha \in[28 \mathrm{deg}, 29 \mathrm{deg}], p \approx-3.5 \mathrm{~s}^{-1}, r \approx-1.9 \mathrm{~s}^{-1}$; and bifurcation diagrams further change in comparison with the thirdorder approximate results. At the same time, highly unstable equilibria at $\alpha=35 \mathrm{deg}$ are practically invariable both to elevator deflection and adding damping terms. In more detail, the influence of the value of the damping term on the range and stability of spin equilibria was analyzed by multiplying all the added damping terms by a factor of $k \in[0,1]$; the results for $k=0,0.5$, and 1 are shown in Fig. 11. The presence of the branch of stable spin means that a jump to spin is possible from a straight level flight by pitching the nose up. In the absence of stable spin (at $k<0.8$ ), there is no equilibrium spin branch to jump to.

Another example of influence of damping terms on spin is presented in Fig. 12, where results of continuation of equilibria on rudder deflection at $\delta_{a}=-1, \delta_{e}=-5,-10,-15$, and $-20 \mathrm{deg}$ are presented. Here, with adding the damping terms, parameters of equilibria are practically invariable but stability properties change significantly. With damping terms, stable steady spins exist at $\delta_{e}=-20 \mathrm{deg}, \quad \delta_{e} \in[-25 \mathrm{deg},-12 \mathrm{deg}], \quad \delta_{e}=-15 \mathrm{deg}, \quad \delta_{e} \in$ [-25 deg, $-18 \mathrm{deg}$ ] (Fig. 12b). Without damping, all steady spins are unstable. This often implies that the spin dynamics is more complicated: perhaps periodic or chaotic. But, in this case, no stable attractors were found. Thus, the correct value of damping terms is important for adequate spin prediction.

\section{B. Sensitivity to Uncertainties of Static Aerodynamic Coefficients}

In the present analysis, the uncertainties of static aerodynamic coefficients are regarded as independent, and various combinations of uncertainties within the bounds presented in Fig. 7 are considered. Calculations show that uncertainties of the aerodynamic load coefficients $\Delta C_{Y}$ and $\Delta C_{Z}$ influence the results of equilibrium calculation very slightly. The scatter of the moment coefficients $\Delta C_{l}, \Delta C_{m}$, and $\Delta C_{n}$ influences the equilibrium parameters much more strongly. The following results are obtained for the aerodynamic model [Eqs. (1) and (2)] (with the nominal damping according to Fig. 4) at the flight altitude of $H=3000 \mathrm{~m}$. Figures 13 and 14 show the sensitivity of equilibrium parameters to variation of static coefficients when all moment coefficients are increased inside the confidence limits: $+\Delta C_{i}(i=l, m, n)$, or decreased $-\Delta C_{i}$, for equilibria with positive $(\Omega>0)$ and negative $(\Omega<0)$ rotation rates. Thin lines (or small markers) correspond to the perturbed coefficients, and thick lines correspond to the nominal coefficients. It can be seen that the bifurcation diagrams, and especially the position of foldover points, essentially depend on the coefficients perturbations; thus, ranges of spin existence can significantly depend on the aerodynamic uncertainties. Stability properties also depend on these uncertainties. At the same time, unstable equilibria at $\alpha=35 \mathrm{deg}$ are insensitive to both the elevator deflection and the uncertainty of the coefficients.

Figure 15 shows sensitivity of the parameters of the same spin equilibria to separate variations of roll, pitch, and yaw coefficients for the branch with $\Omega>0$. It can be seen that, for these spins, the roll uncertainty has the largest influence on spin parameters. The yaw uncertainty results in less shift of foldover points but can lead to the appearance of stable spin.

Figure 16 presents the worst-case combinations of uncertainties of aerodynamic coefficients for spins with $\Omega>0$ (Fig. 16a) and spins with $\Omega<0$ (Fig. 16b). The worst case means, in this case, the largest difference with the nominal case: the roll and yaw uncertainties of different signs usually result in the largest difference in spin parameters compared with the nominal aerodynamic coefficients.

Despite the diversity of spin parameters under aerodynamic uncertainties, for the considered aircraft configuration, all the considered spins (stable and unstable) are recoverable by moving the elevator to the neutral position.

Since a picture of spins significantly depends on the inertia parameters, some examples of spin were additionally calculated for the considered aircraft with minimal fuel: $m=870 \mathrm{~kg}, I_{x}=$ $1185 \mathrm{~kg} \cdot \mathrm{m}^{2}, I_{y}=2195 \mathrm{~kg} \cdot \mathrm{m}^{2}, I_{z}=2636 \mathrm{~kg} \cdot \mathrm{m}^{2}$, and centerof-gravity position of $0.16 \bar{c}$. Figure 17 shows branches of equilibria with $\Omega>0$ for the nominal and the worst-case combinations of uncertainties $\left(-C_{l},+\Delta C_{m},+\Delta C_{n}\right)$ at $\delta_{a}=0.5$, and $\delta_{r}=0$. A range of spin equilibria from $\delta_{e}=[-20 \mathrm{deg},-17 \mathrm{deg}]$ for the nominal coefficients expands to the full range of elevator deflections 

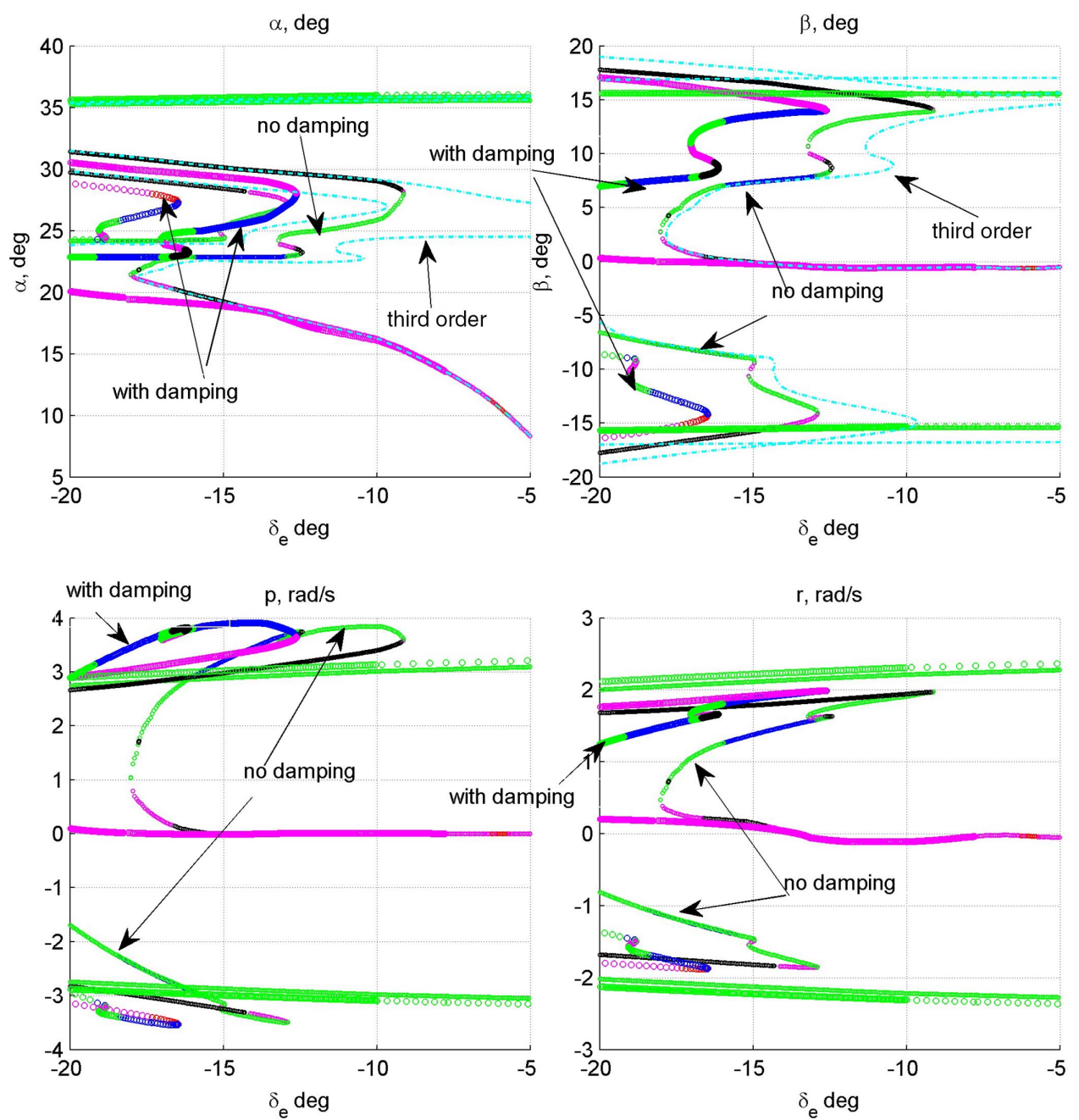

Fig. 10 Comparison of equilibrium parameters with and without damping terms: $H=3000 \mathrm{~m}, \delta_{a}=0$, and $\delta_{r}=0$.
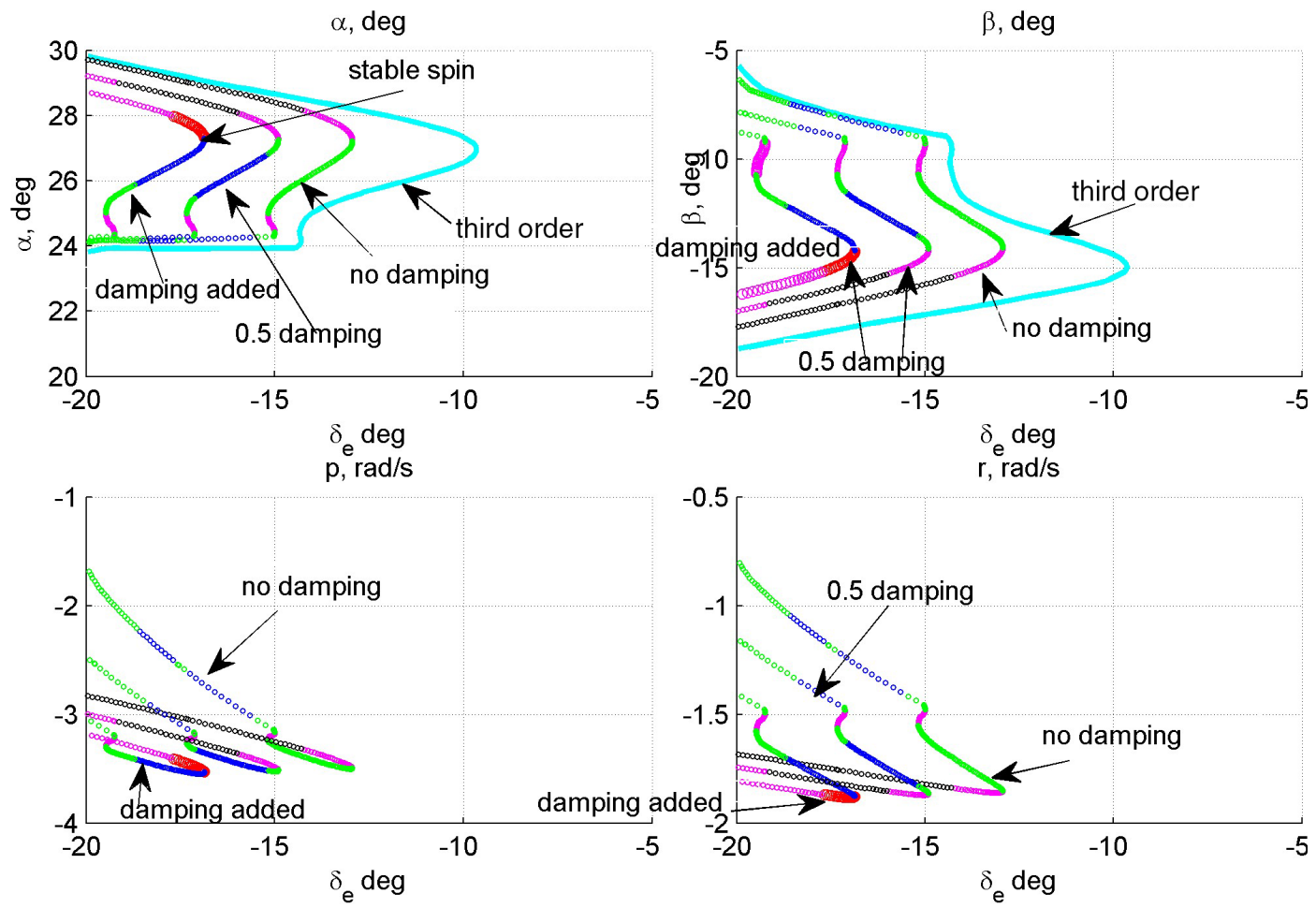

Fig. 11 Influence of value of damping terms: $H=3000 \mathrm{~m}, \delta_{a}=0$, and $\delta_{r}=0$. 

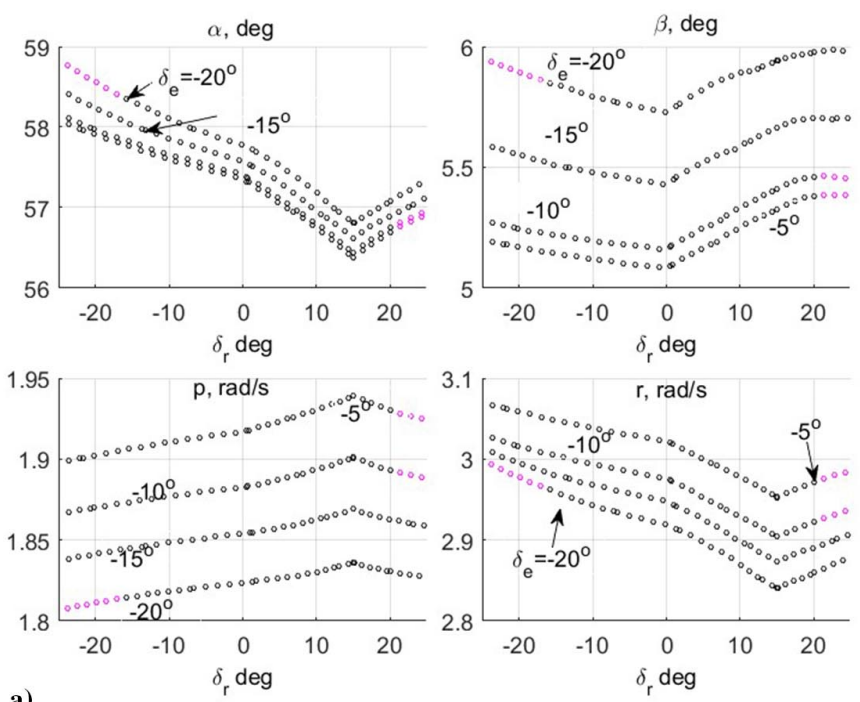

a)
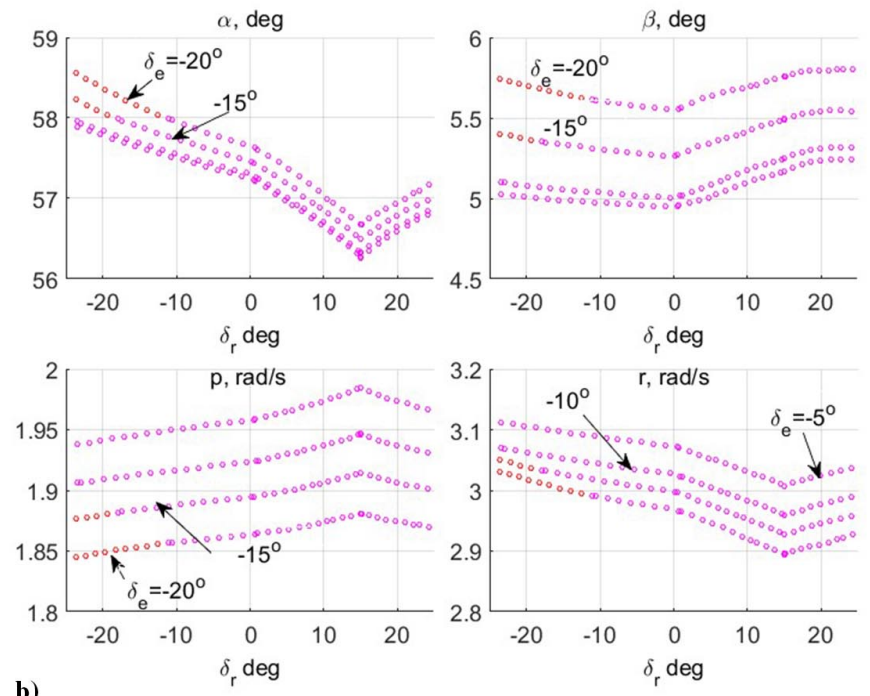

b) damping.

[-20 deg, $5 \mathrm{deg}]$ in the worst case of uncertainties, and the range of stable spin equilibria appears at $\delta_{e}=[-20 \mathrm{deg},-19 \mathrm{deg}]$ and $\alpha=55 \mathrm{deg}$. Figure 17 also shows amplitudes of stable oscillatory spins originating in the result of the loss of stability of steady solutions (a Hopf bifurcation point) outside the range of $\delta_{e}$ values. For the worst-case perturbation $\left(-\Delta C_{l},+\Delta C_{m},+\Delta C_{n}\right)$, periodic solutions transform into quasi-periodic and chaotic spin attractors. This takes place in a narrow range $-4 \mathrm{deg}<\delta_{e}<-3 \mathrm{deg}$ as a result of subsequent bifurcations. Examples of stable periodic and quasiperiodic trajectories and their projections onto the $(\alpha, \beta)$ plane are shown in Fig. 18. For the nominal model, the range of stable oscillatory spins is very small: $\delta_{e}=[-20 \mathrm{deg},-19.5 \mathrm{deg}]$. Such a differ- ence can be explained, perhaps, by the fact that stable spin takes place in this case in the range of highest lateral aerodynamic asymmetry. Fortunately, in both cases, spins are recoverable by setting all the controls to the neutral position.

Two other examples are shown Fig. 19, where continuation of spin solutions on aileron deflection is performed. The dependence of the parameters of stationary and approximate amplitudes of oscillatory chaotic spins for the nominal and perturbed yaw coefficients $-\Delta C_{n}$ is shown in Fig. 17a for $\delta_{e}=-15 \mathrm{deg}, \delta_{r}=0$, and $\Omega<0$. It can be seen that the ranges of stable spins are different for the nominal and perturbed coefficients, and this makes a problem for adequate prediction of the aircraft behavior after loss of control. A good thing is
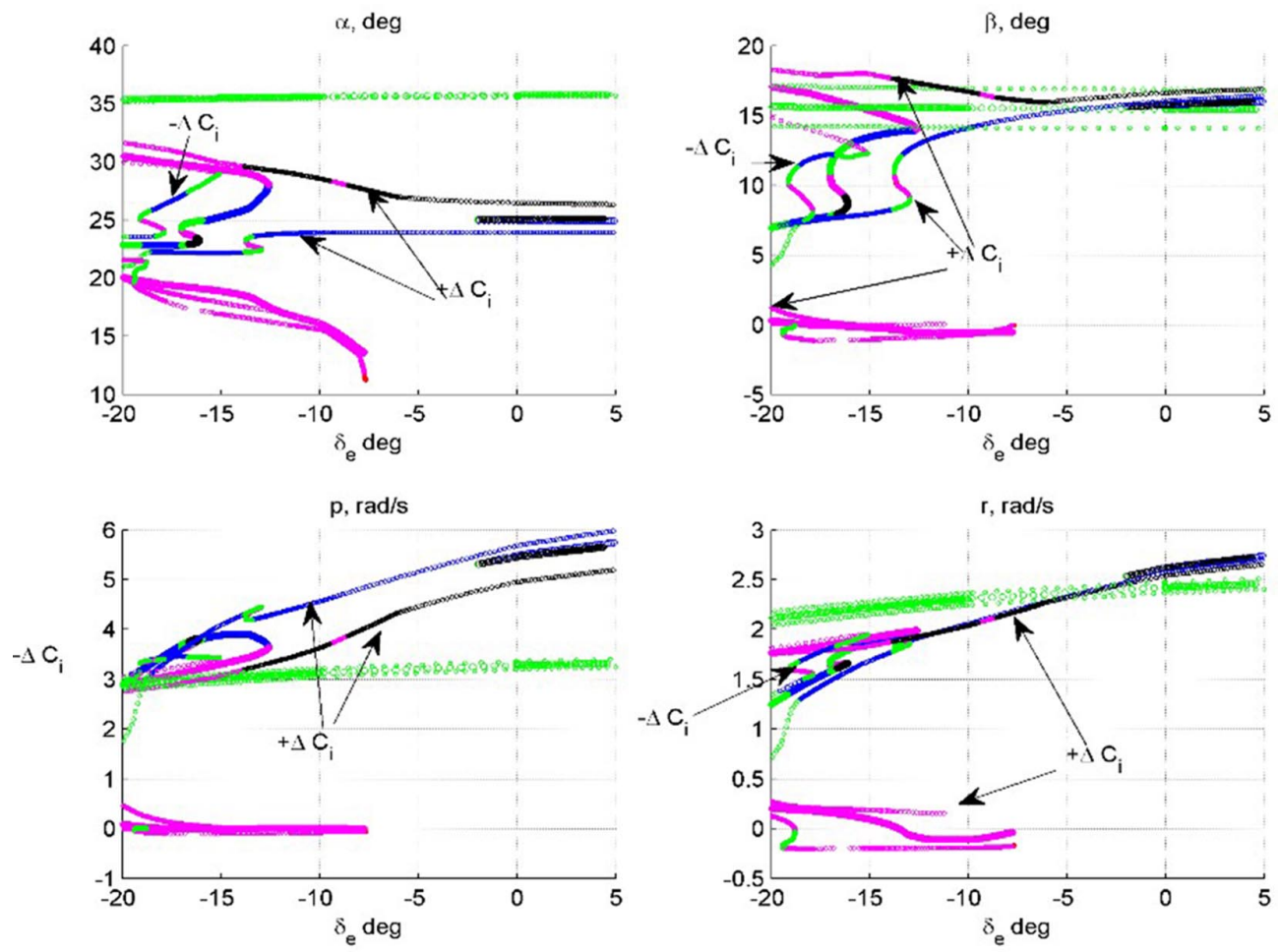

Fig. 13 Sensitivity of spin parameters to variation of static coefficients: $\Omega>0$. 

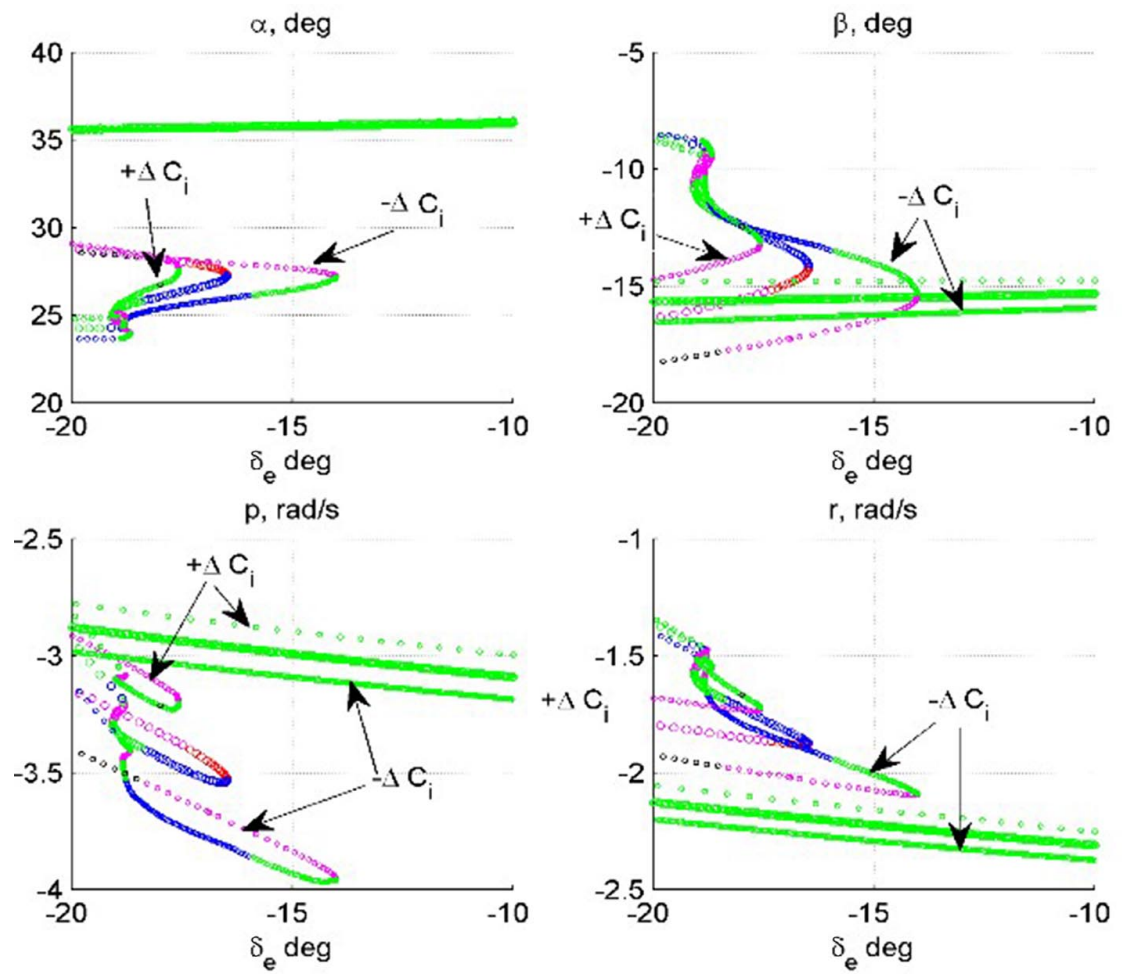

Fig. 14 Sensitivity of spin parameters to variation of static coefficients: $\Omega<0$.

that, in both cases, there are no stable spins at $\delta_{a}=0$; that is, in both cases, spins are recoverable by setting the aileron to the neutral position. Figure $17 \mathrm{~b}$ shows the branches of steady spins and the ranges of stable periodic and chaotic spins for the nominal and worst-case uncertainties of the aerodynamic coefficients $\left(-\Delta C_{l}\right.$,
$+\Delta C_{m}$, and $+\Delta C_{n}$ ) for $\Omega>0$. For the worst-case perturbation of the aerodynamic coefficients, the range of the stable oscillatory spins is much wider than for the nominal coefficients.

Thus, the regions of spin (in particular, stable spin) can noticeably differ with minor variations of the aerodynamic coefficients.
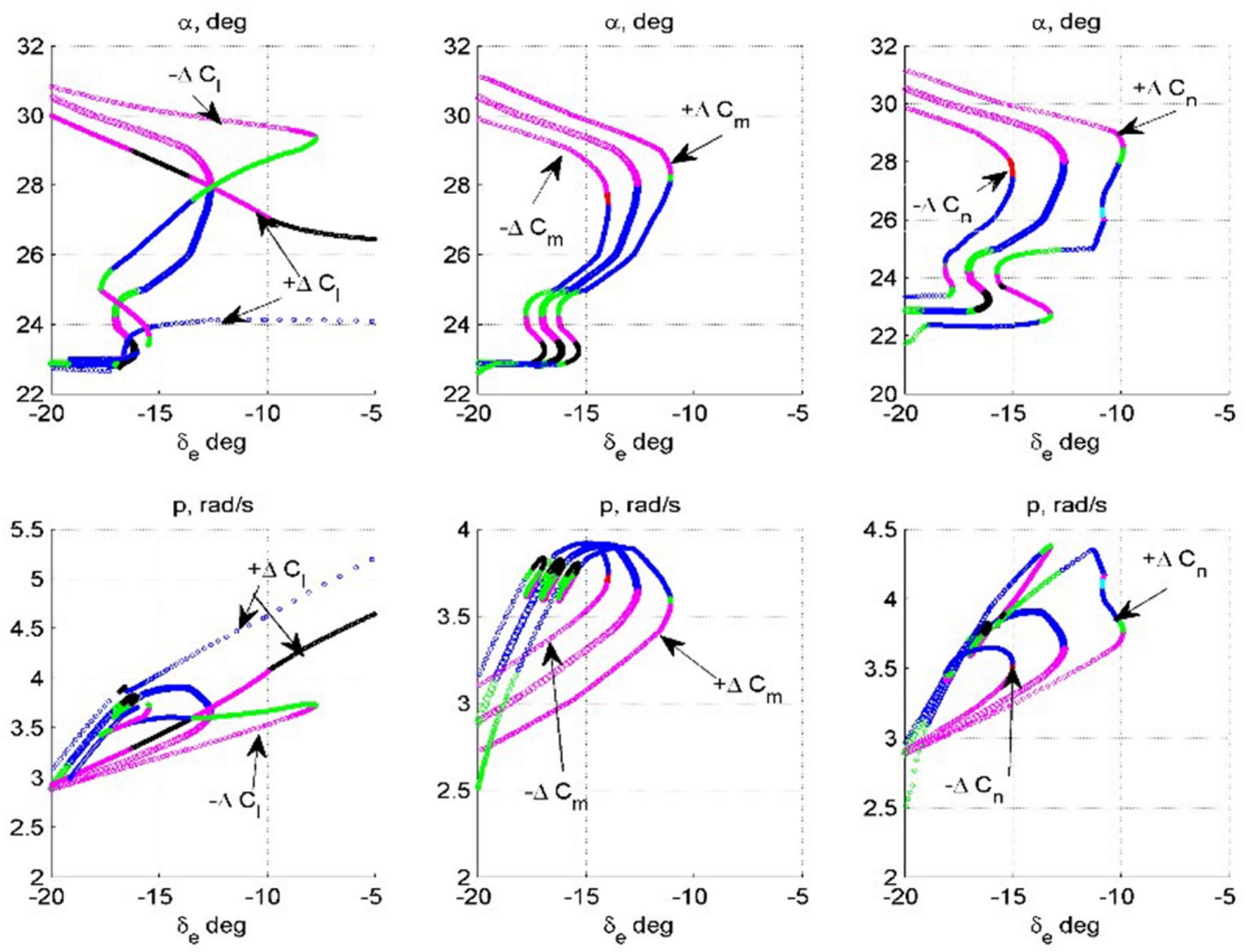

Fig. 15 Sensitivity of spin parameters to separate variations of moment coefficients. 

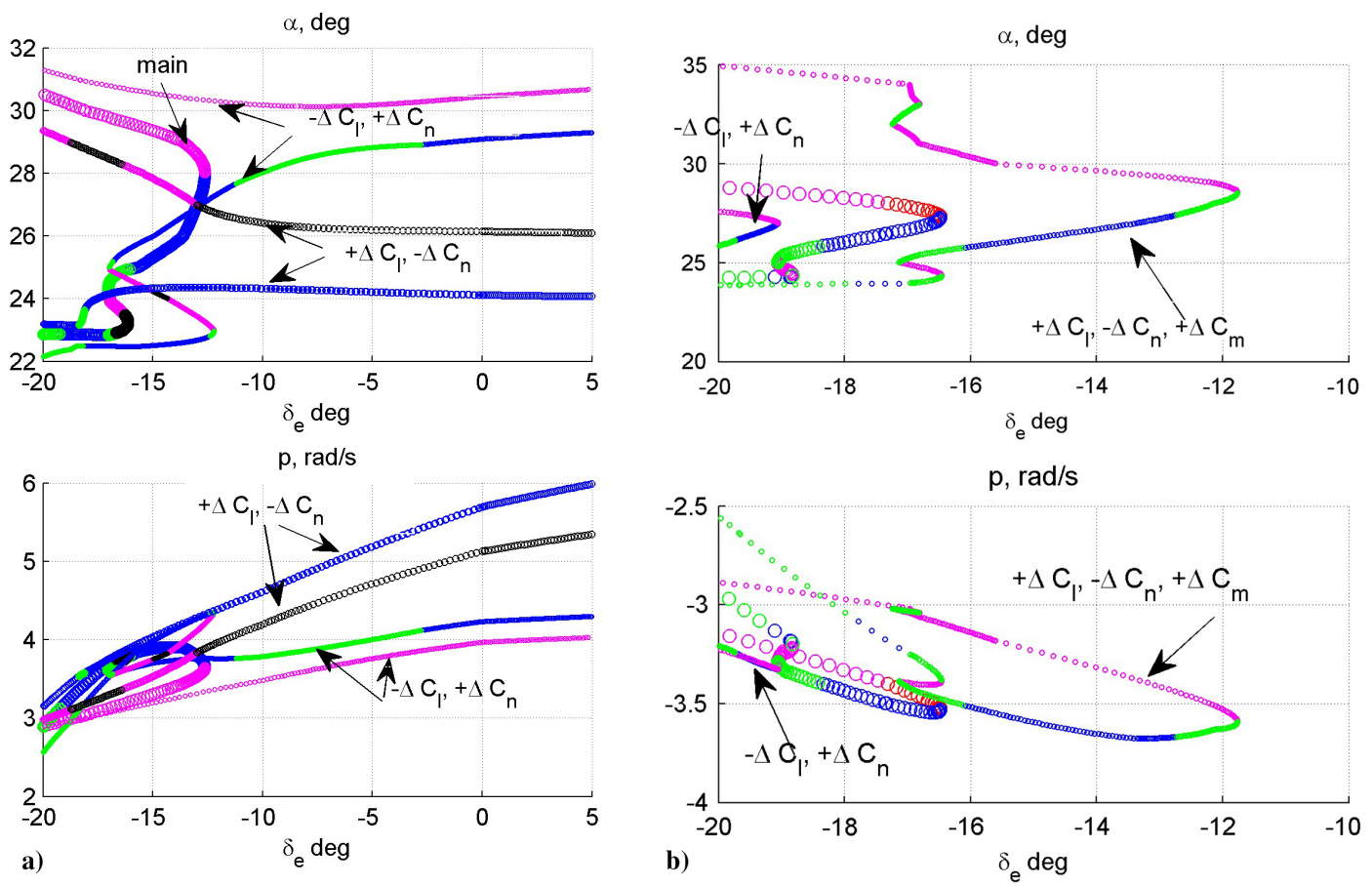

Fig. 16 Worst-case variations of aerodynamic coefficients: a) $\Omega>0$, and b) $\Omega<0$.

Spin trajectories substantially depend on the values of the damping terms that, as all experimental data, have uncertainties. Figure 20 shows the change in the spin trajectories with an increase in damping by $15 \%$ compared to the nominal case: chaotic trajectories become periodical. With decreasing damping, the attracting set (consisting of chaotic or periodic trajectories) can turn into weakly unstable or quasi stable, which is much more difficult to calculate than the stable steady modes, but which can also be very dangerous. This fact should be taken into account when determining dangerous spins.
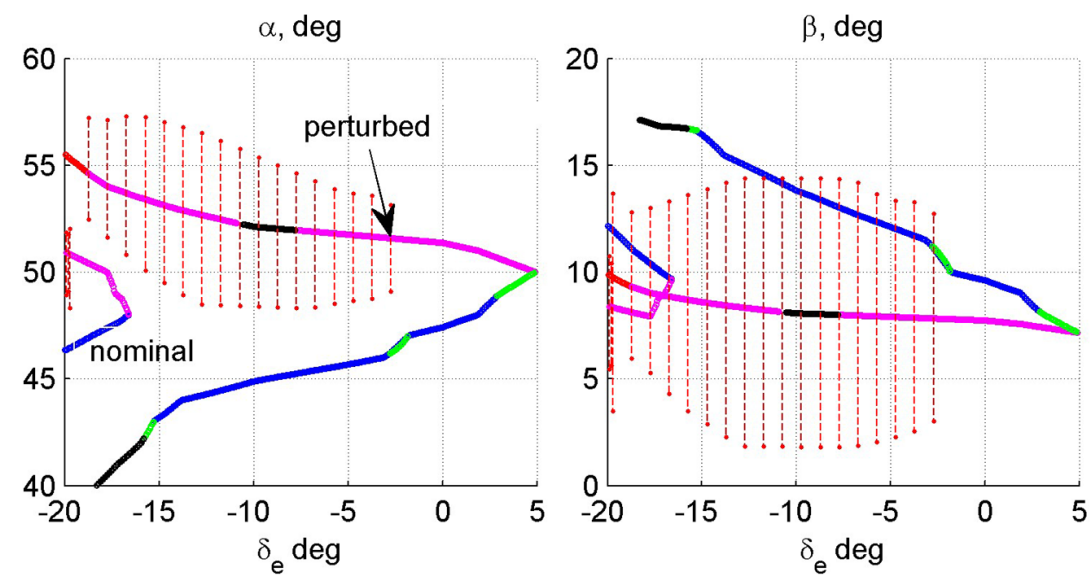

$\mathrm{p}, \mathrm{rad} / \mathrm{s}$
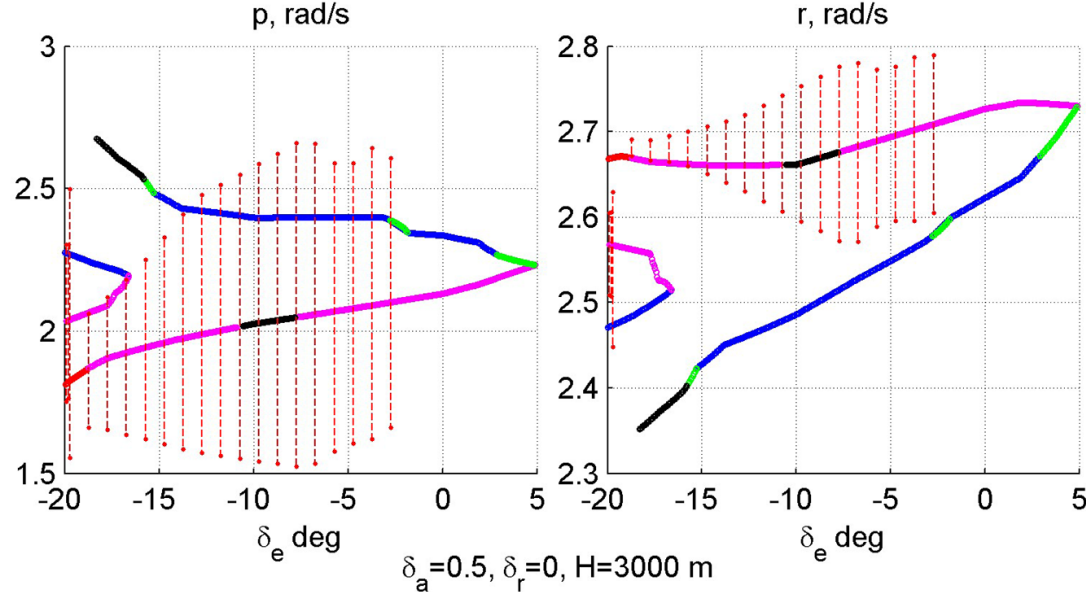

Fig. 17 Stable oscillatory spins for nominal and perturbed aerodynamic coefficients. 

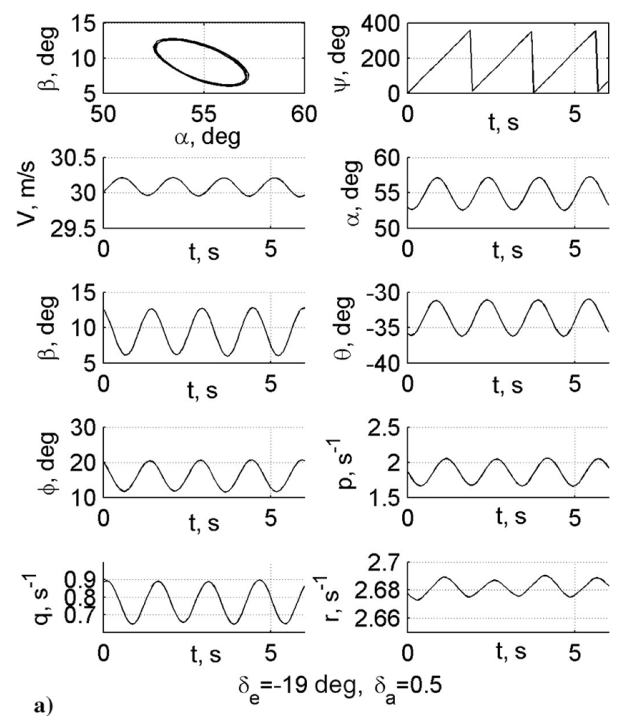
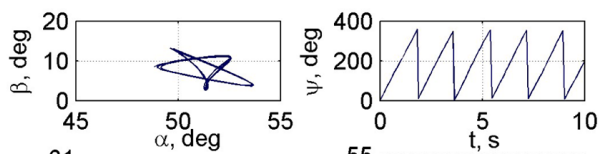

$>{ }_{30.6}^{30.8} \underbrace{31}_{\mathrm{t}, \mathrm{s}}$
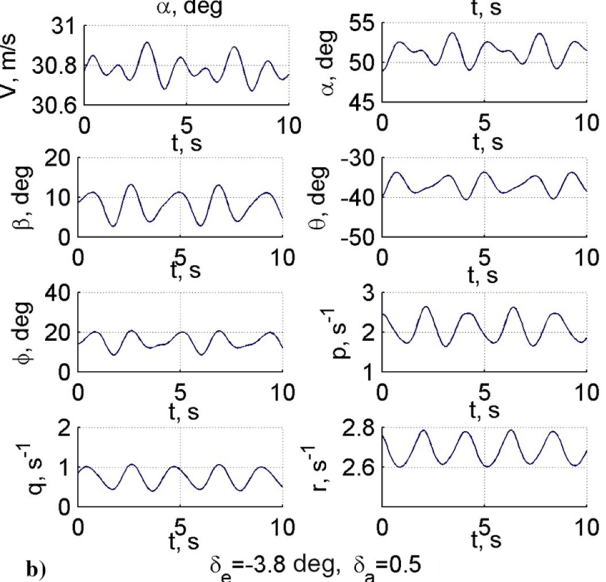

Fig. 18 Examples of trajectories of stable oscillatory spins.

\section{Sensitivity of Flat Spin to Aerodynamic Uncertainties}

For the nominal mass/inertia configuration of the aircraft (Sec. II.A), two separate branches of flat spins were also found at $\delta_{a}=\overline{0}$ and $\delta_{r}=0$ at high angles of attack (65-70 deg). They are shown in Fig. 21, which is an extension of Fig. 8 for high angles of attack. Branches of oscillatory unstable equilibria (magenta solid lines at $\alpha \approx 65 \mathrm{deg}$ ) and highly unstable equilibria (green dotted lines at $\alpha \approx 70 \mathrm{deg}$ ) exist only at $\Omega>0$.

Oscillatory unstable steady-state spins give branches of stable oscillatory spins. Oscillatory spins are periodic at $\Omega>0$; and they are periodic or chaotic, depending on elevator deflection, at $\Omega<0$. Amplitudes of periodic spins and approximate amplitudes of chaotic spins, along with other steady-state solutions (both red and magenta vertical lines correspond to stable oscillatory spins) are shown in Fig. 22. Examples of trajectories are shown in Fig. 23.

An important feature of these flat spin modes is their low sensitivity: both to deflections of control surfaces (the efficiency of controls at high angles of attack is small) and to uncertainties of the aerodynamic coefficients. Figure 24 demonstrates this low sensitivity of spin equilibria to the yaw moment uncertainty. The sensitivity is also low for the oscillatory spins.

An example of high sensitivity of bifurcation diagrams to yaw coefficient uncertainty is shown in Fig. 25. The uncertainty varies from 20 to $70 \%$ of the maximum yaw moment uncertainty $+\Delta C_{n}$. It can be seen that the bifurcation picture changes intricately. However, the most dangerous, flat unrecoverable spins with large
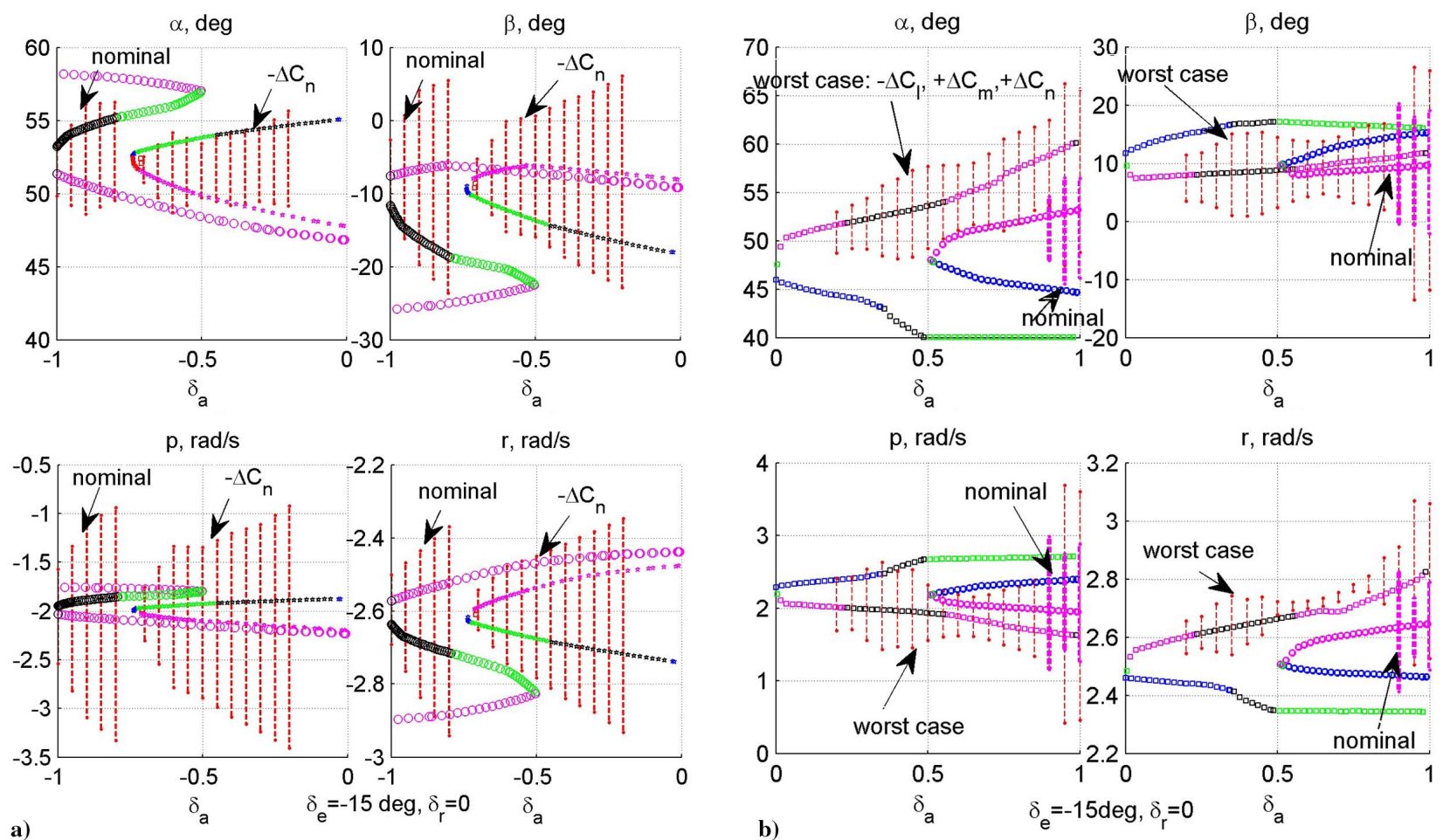

Fig. 19 Parameters of stationary and oscillatory spins for the nominal and perturbed coefficients. 

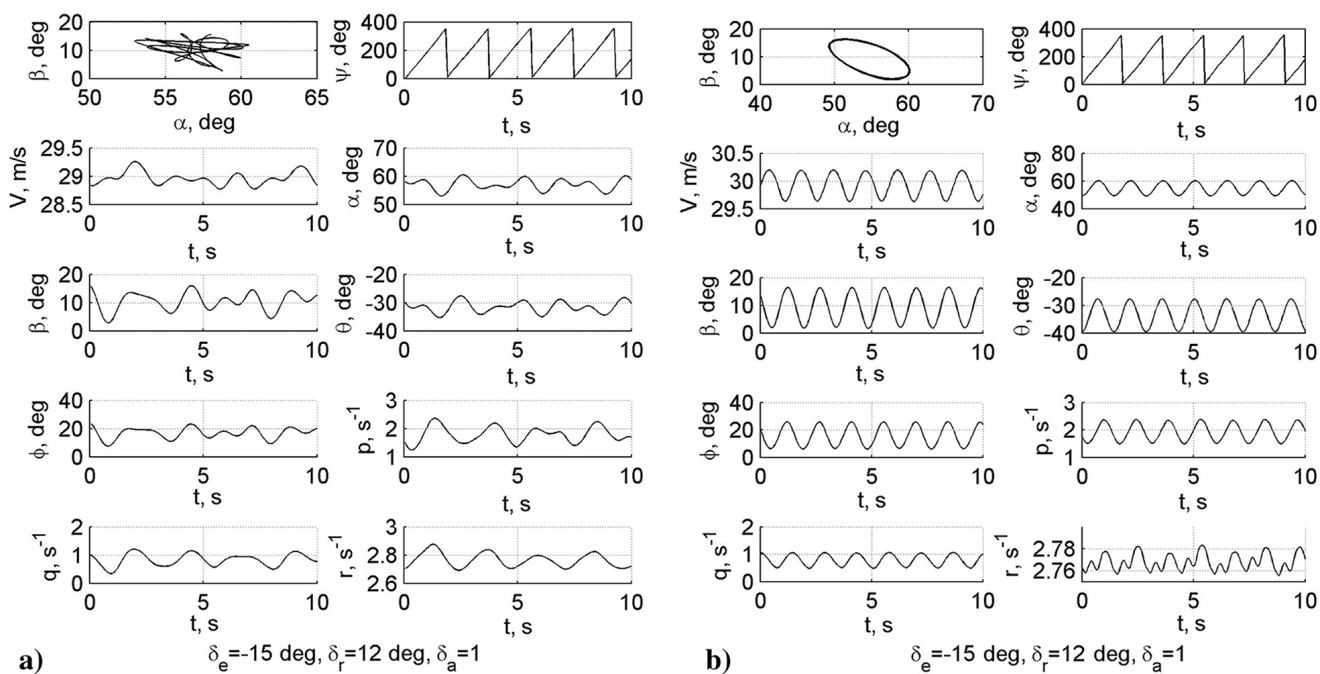

ס 400

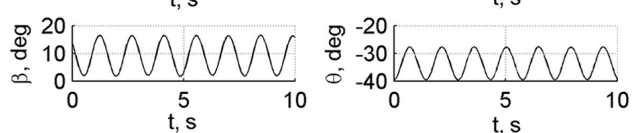

$\underbrace{2}_{0}{ }_{0}^{20}$

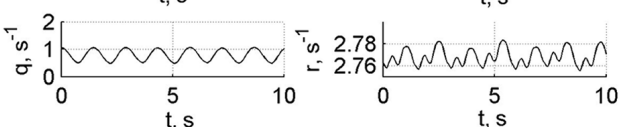

b) $\delta_{\mathrm{e}}=-15 \mathrm{deg}, \delta_{\mathrm{r}}=12 \mathrm{deg}, \delta_{\mathrm{a}}=1$

Fig. 20 Sensitivity of trajectories of stable spin to variation of damping terms of the aerodynamic model: a) nominal damping (chaotic spin), and b) damping increased by $15 \%$ (periodic spin).
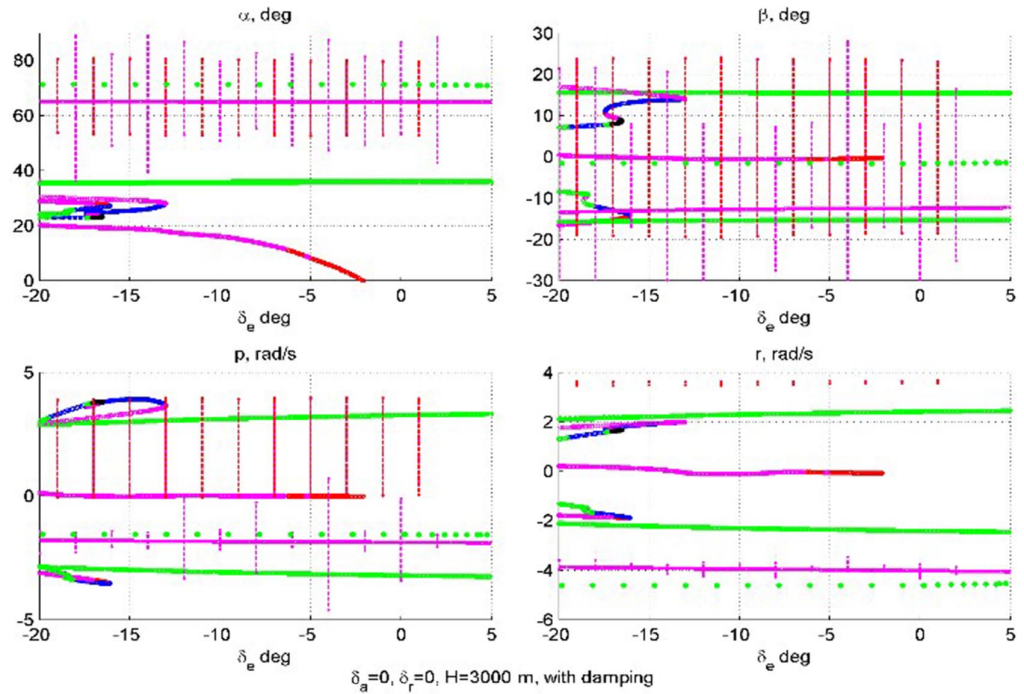

Fig. 21 Steady-state and stable oscillatory spins depending on elevator deflection: $\delta_{a}=0$, and $\delta_{r}=0$.
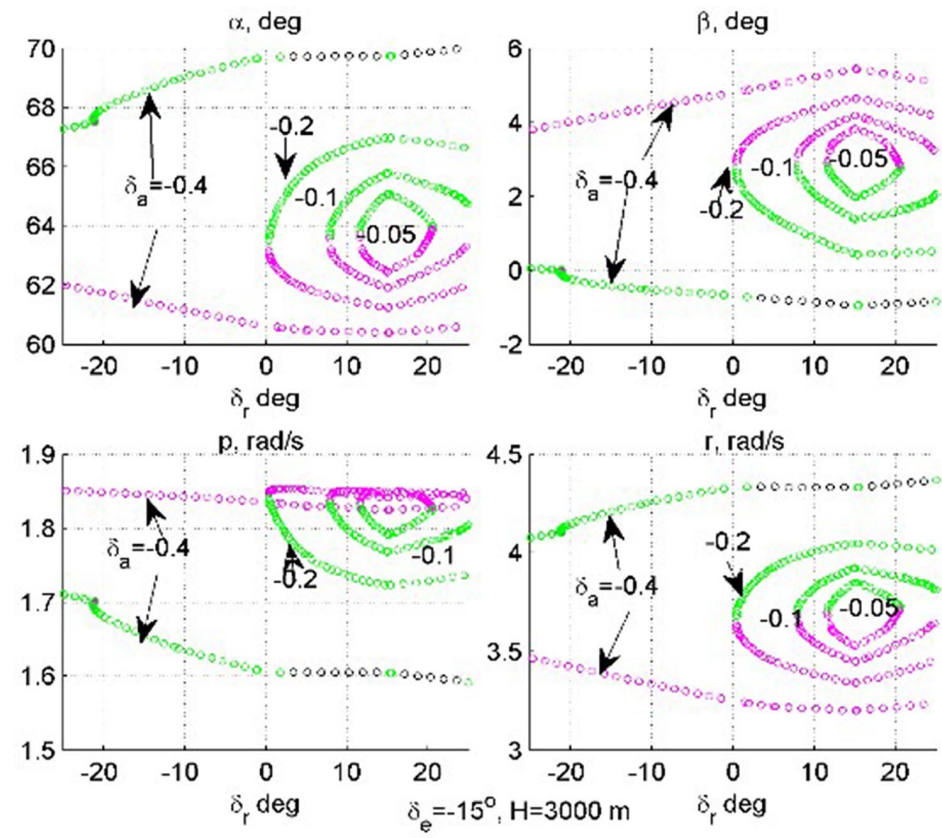

Fig. 22 Flat spin dependence on aileron and elevator deflection $(\Omega>0)$. 

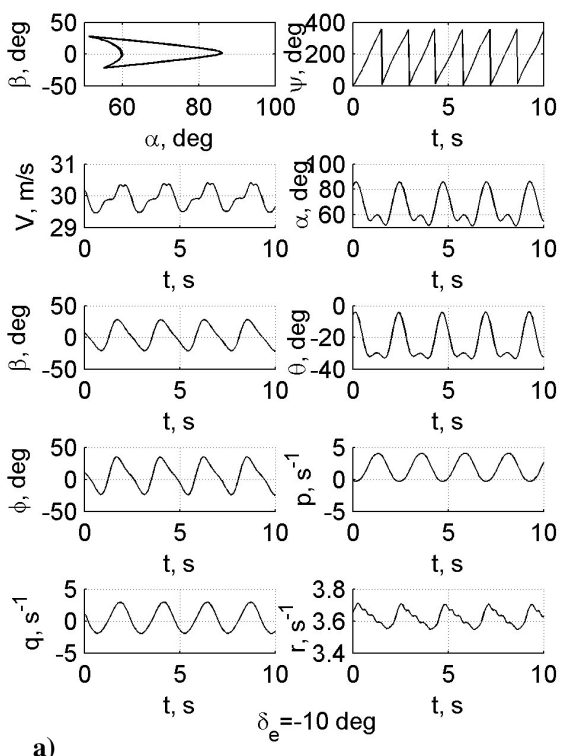
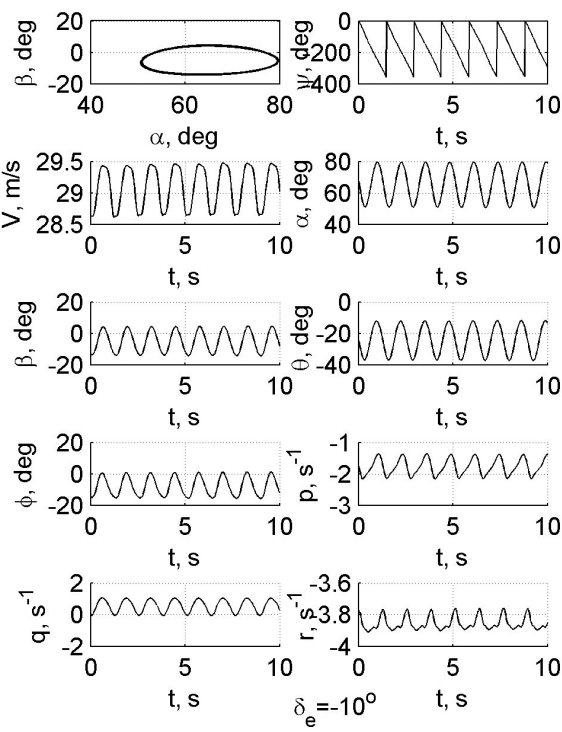

b)
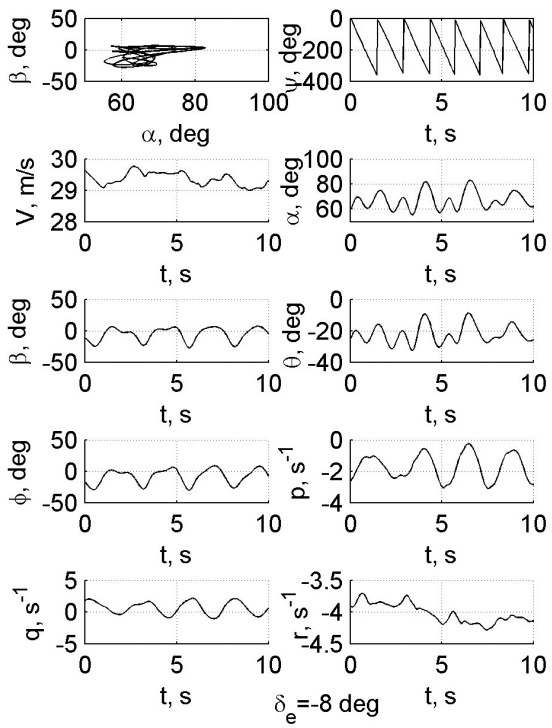

c)

Fig. 23 Flat spin trajectories: a) $\Omega>0, \delta_{e}=-10 \mathrm{deg}$; b) $\Omega<0, \delta_{e}=-10 \mathrm{deg}$; c) $\Omega<0, \delta_{e}=-8 \mathrm{deg}$. regions of capture are significantly less sensitive to aerodynamic uncertainties. It would be useful to estimate the size of the attraction region of stable spin. An attempt to make such an estimate for stationary spins was done in Ref. [20]. Here, and in many other cases, the most dangerous stable spin modes are oscillatory; and the estimation of their attraction regions, as well as understanding its correlation with sensitivity to uncertainties, is problematic.

\section{Spin Entry/Recovery: Sensitivity to Uncertainties}

In practice, it is important to consider those spin modes in which the aircraft can stall from normal flight. In this analysis, spin entry is performed from a level, symmetric trim flight at an altitude of
$H=3000 \mathrm{~m}$ by elevator deflection up to $\delta_{e}=-15-20 \mathrm{deg}$ and rudder deflection up to $\delta_{r}= \pm 20$ and $25 \mathrm{deg}\left(\delta_{a}=0\right)$ for a few seconds. The quick reaction of the pilot allows us to recover the aircraft from spin, if the angle of attack has not yet reached $\sim 40 \mathrm{deg}$. This is possible by setting the controls in a neutral positions in both cases, for the nominal, and perturbed aerodynamic models. The delay in setting the controls to the neutral position results in flat spin, which is not recoverable. A similar behavior is observed for the nominal and perturbed aerodynamic models. Using the aileron and rudder against rotation sometimes allows us to recover the aircraft from a flat spin, but not for all perturbations of the aerodynamic model; one example is shown in Fig. 26. In any case, the pilot's actions must be very accurate; otherwise, the aircraft may enter another spin with the opposite rotation.
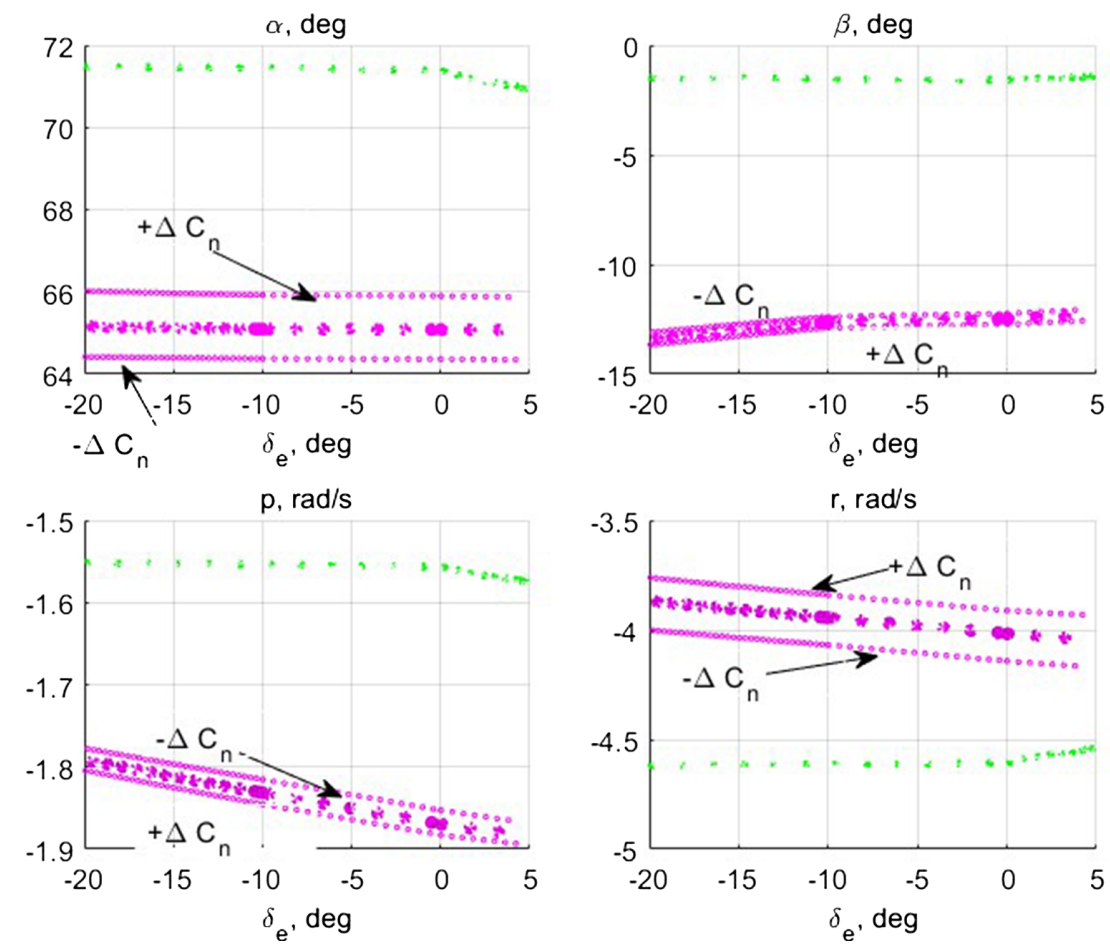

Fig. 24 Sensitivity of spin equilibria to yaw moment uncertainty $(\Omega<0)$. 

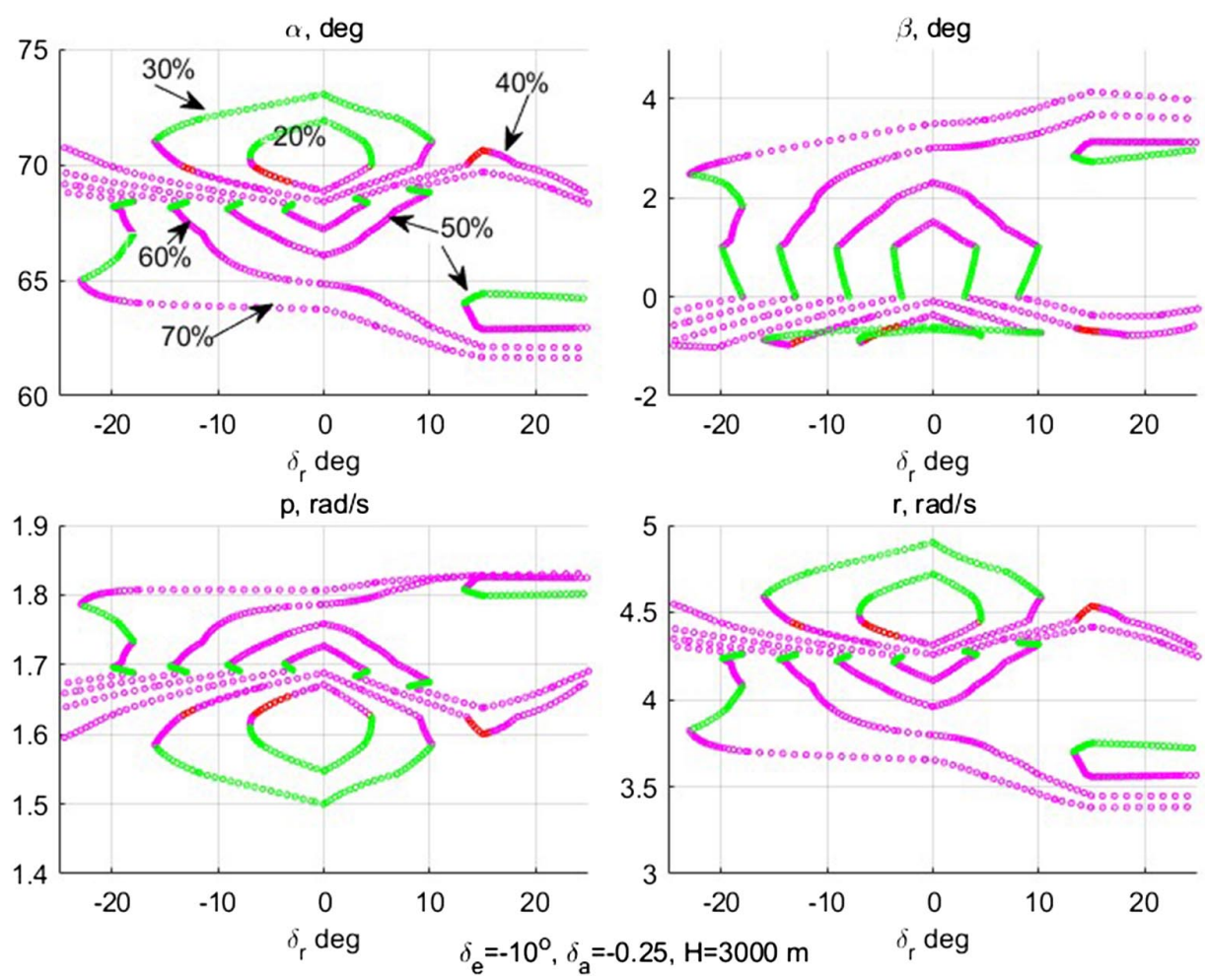

Fig. 25 Example of sensitivity of bifurcation diagrams to uncertainties of yaw moment: $\delta_{e}=-10$, and $\delta_{a}=-0.25$.
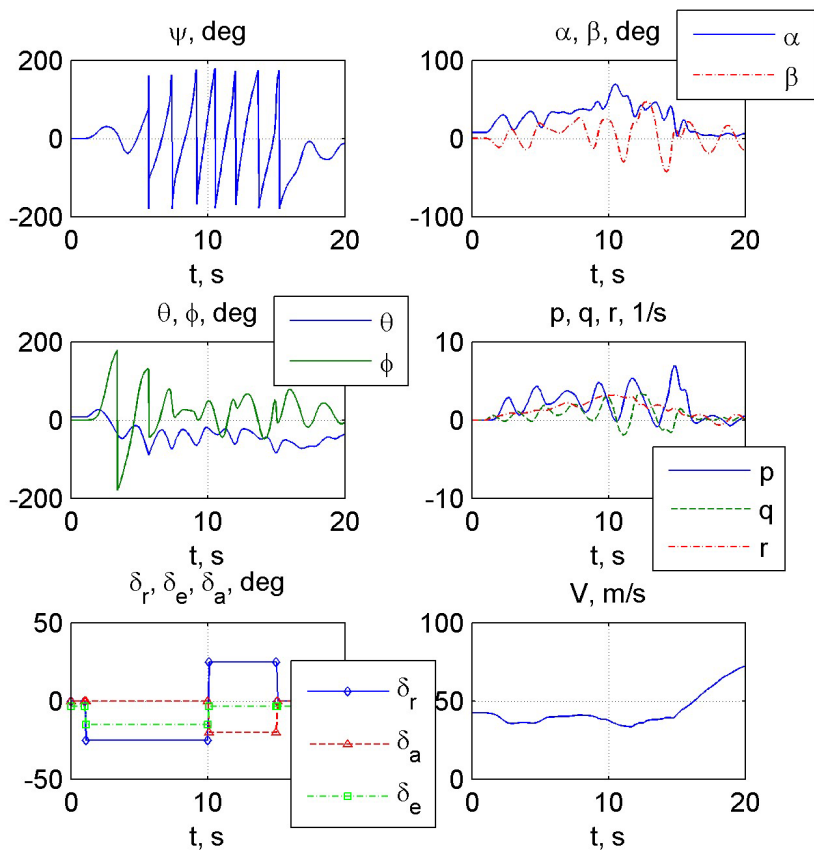

$\mathrm{V}, \mathrm{m} / \mathrm{s}$

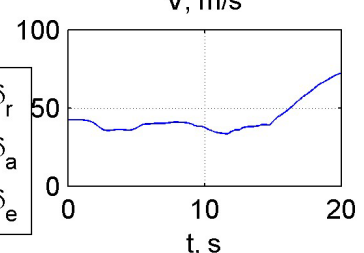

$\mathrm{H}, \mathrm{m}$
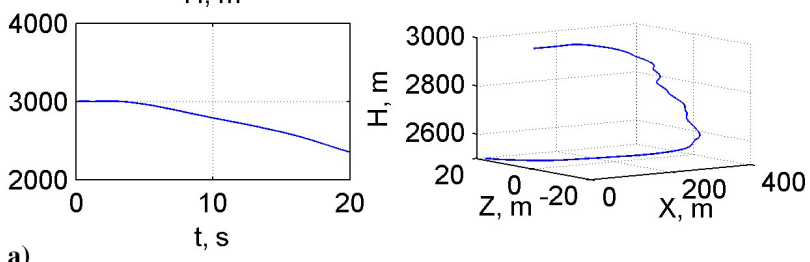
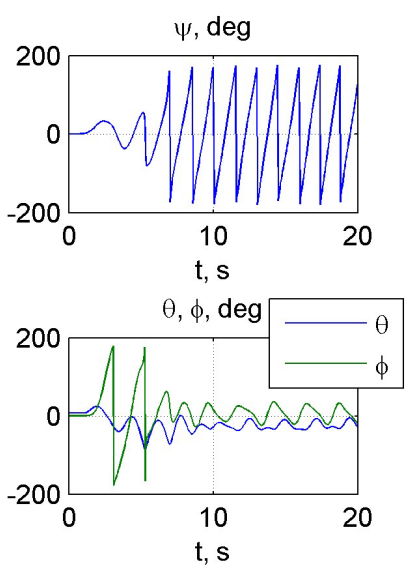

$\delta_{r}, \delta_{e}, \delta_{a}, \operatorname{deg}$

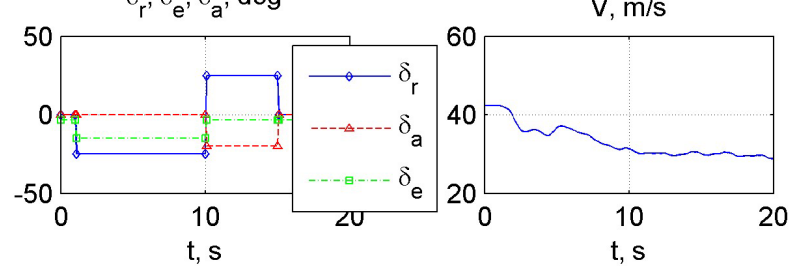

$\mathrm{H}, \mathrm{m}$

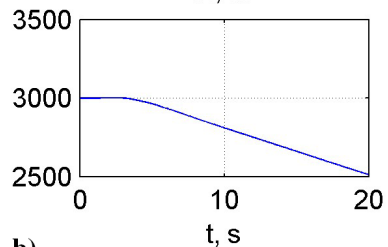

b)

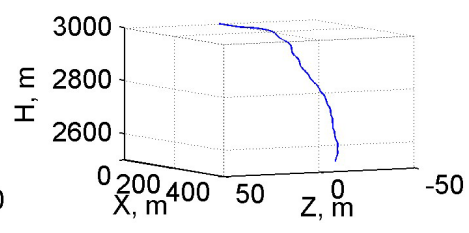

Fig. 26 Spin entry and recovery: a) nominal aerodynamics (recovery), and b) perturbed $+\Delta C_{n}($ no recovery). 


\section{Conclusions}

A representative aerodynamic model of a light aircraft was developed in a wide range of angles of attack, sideslip, angular rates, and control deflections; this model was supplemented by the uncertainties, which were obtained based on a large number of experiments. A comparative analysis of the spin parameters, as well as the spin entry/recovery behavior for the nominal and perturbed aerodynamic models, revealed the high sensitivity of the bifurcation diagrams to the uncertainties of the aerodynamic coefficients: especially the roll and yaw moment coefficients, as well as the lower sensitivity of the most dangerous stable flat spins to aerodynamic uncertainties. The desirability of spin analysis has become apparent, not only for the nominal aerodynamic model but also for a model that takes into account aerodynamic uncertainties: especially the yaw moment uncertainty.

\section{Acknowledgments}

The authors are obliged to the ONERA-TsAGI collaborative project "Improvement of Representativeness of Models" for the opportunity to perform this work. The authors express sincere gratitude to the anonymous reviewers for their helpful comments and valuable suggestions.

\section{References}

[1] Balas, G., Chiang, R., Packard, A., and Safonov, M., Robust Control Toolbox, User's Guide, MathWorks, Natick, MA, 2020, http://www .mathworks.com/help/pdf_doc/robust/robust_ug.pdf [retrieved 26 May 2020].

[2] Carroll, J. V., and Mehra, R. K., "Bifurcation Analysis of Nonlinear Aircraft Dynamics," Journal of Guidance, Control, and Dynamics, Vol. 5, No. 5, 1982, pp. 529-536. https://doi.org/10.2514/3.56198

[3] Jahnke, C. C., and Culick, F. E. C., "Application of Bifurcation Theory to the High-Angle-of-Attack Dynamics of the F-14," Journal of Aircraft, Vol. 31, No. 1, 1994, pp. 26-34.

https://doi.org/10.2514/3.46451

[4] Goman, M. G., and Khramtsovsky, A. V., "Application of Continuation and Bifurcation Methods to the Design of Control Systems," Philosophical Transactions of the Royal Society of London, Series A: Mathematical, Physical, and Engineering Sciences, Vol. 356, No. 1745, 1998, pp. 2277-2295. https://doi.org/10.1098/rsta.1998.0274

[5] Goman, M.G., Zagaynov, G.I., and Khramtsovsky, A.V., "Application of Bifurcation Methods to Nonlinear Flight Dynamics Problems," Progress in Aerospace Sciences, Vol. 33, No. 59, 1997, pp. 539-586. https://doi.org/10.1016/S0376-0421(97)00001-8

[6] Guicheteau, P., "Bifurcation Theory: A Tool for Nonlinear Flight Dynamics," Philosophical Transactions of the Royal Society of London, Series A: Mathematical, Physical, and Engineering Sciences, Vol. 356, No. 1745, 1998, pp.2181-2201.

[7] Lowenberg, M. H., "Bifurcation Analysis of Multiple-Attractor Flight Dynamics," Philosophical Transactions of the Royal Society of London, Series A: Mathematical, Physical, and Engineering Sciences, Vol. 356,
No. 1745,1998 , pp. $2297-2319$.

https://doi.org/10.1098/rsta.1998.0275

[8] Aerodynamics, Stability and Controllability of Supersonic Aircraft, edited by Bushgens, G., and Nauka, S., Fizmatlit, Moscow, 1998, pp. 1-816 (in Russian).

[9] Khrabrov, A., Sidoryuk, M., and Goman, M., "Aerodynamic Model Development and Simulation of Airliner Spin for Upset Recovery," Progress in Flight Physics, Vol. 5, European Conference for Aerospace Sciences Book Series, edited by Ph. Reijasse, D. Knight, M. Ivanov, and I. Lipatov, Torus Press, Moscow, 2013, pp. 621-636. https://doi.org/10.1051/eucass/201305621

[10] Farcy, D., Khrabrov, A., and Sidoryuk, M., "Sensitivity of Spin Parameters to Uncertainties of the Aerodynamic Model of a Light Aircraft," Proceedings of the 8th European Conference for Aerospace Sciences (EUCASS), Paper EUCASS2019-537, 2019, https://www.eucass. eu/index.php/conferences-and-publications/eucass-2019 [retrieved 26 May 2020]. https://doi.org/10.13009/EUCASS2019-537

[11] Farcy, D., Khrabrov, A., and Sidoryuk, M., "Investigation of Spins and their Sensitivity to Parameter Variations for the ONERA Aerodynamic Model," 16th ONERA-TsAGI Seminar, ONERA, Palaiseau, France, Oct. 2017, pp. 27-28.

[12] Kalviste, J., "Use of Rotary Balance and Forced Oscillation Test Data in a Six Degrees of Freedom Simulation," AIAA 9th Atmospheric Flight Mechanics Conference, AIAA Paper 1982-1364, 1982.

[13] Murch, A. M., and Foster, J. V., "Recent NASA Research on Aerodynamic Modeling of Post-Stall and Spin Dynamics of Large Transport Airplanes," 45th AIAA Aerospace Sciences Meeting and Exhibit, AIAA Paper 2007-0463, 2007.

[14] Abramov, N. B., Goman, M. G., Khrabrov, A. N., and Soemarwoto, B. I., "Aerodynamic Modeling for Poststall Flight Simulation of a Transport Airplane," Journal of Aircraft, Vol. 56, No. 4, 2019, pp. 1427-1440. https://doi.org/10.2514/1.C034790

[15] Sigaud, O., Salaün, C., and Padois, V., "On-Line Regression Algorithms for Learning Mechanical Models of Robots: A Survey," Robotics and Autonomous Systems, Vol. 59, No. 12, 2011, pp.1115-1129. https://doi.org/10.1016/j.robot.2011.07.006

[16] Vijayakumar, S., D'Souza, A., and Schaal, S., "Incremental Online Learning in High Dimensions," Neural Computation, Vol. 17, No. 12, 2005, pp. 2602-2634. https://doi.org/10.1162/089976605774320557

[17] Schaal, S., and Atkeson, C., "Constructive Incremental Learning from Only Local Information," Neural Computation, Vol. 10, No. 8, 1998, pp. 2047-2084. https://doi.org/10.1162/089976698300016963

[18] Weissman, R., "Status of Design Criteria for Predicting Departure Characteristics and Spin Susceptibility," Journal of Aircraft, Vol. 12, No. 12, 1975, pp. 989-993. https://doi.org/10.2514/3.59904

[19] Paranjape, A., and Ananthkrishnan, N., "Analytical Criterion for Aircraft Spin Susceptibility," Journal of Aircraft, Vol. 47, No. 5, pp. 1804-1807. https://doi.org/10.2514/1.C031010

[20] Sidoryuk, M., and Khrabrov, A., "Estimation of Regions of Attraction of Aircraft Spin Modes," Journal of Aircraft, Vol. 56, No. 1, 2019, pp. 205-216. https://doi.org/10.2514/1.C034936 